UCID- 20995

\title{
Extended Analysis of Constant-Height Hydraulic Fractures for the Estimation of In-Situ Crack-Opening Modulus from Bottomhole Pressure Records
}

\author{
Ananda M. Wijesinghe
}

Prepared for

Morgantown Energy Technology Center

Morgantown, WV

March, 1987

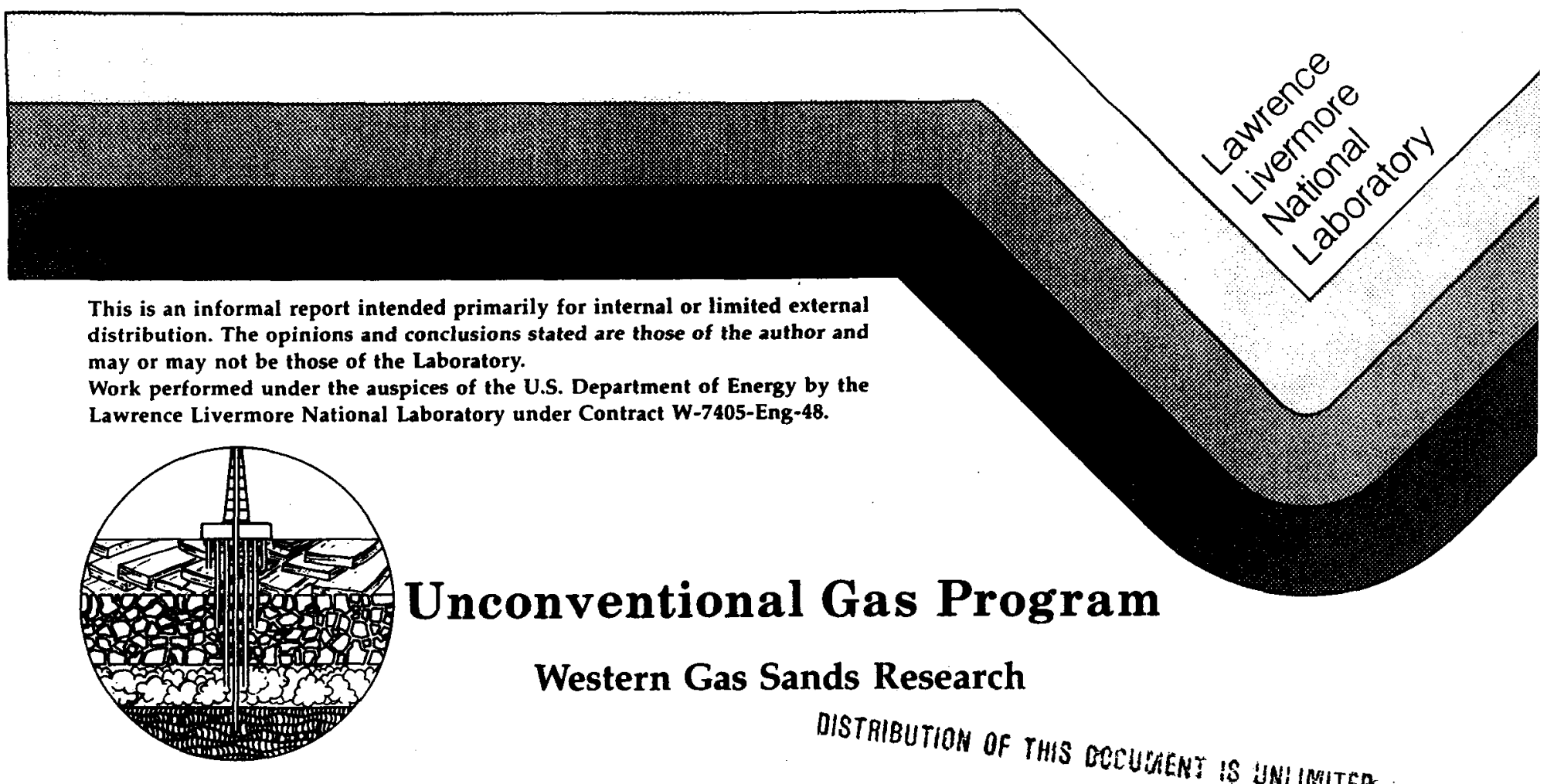


This document was prepared as an account of work sponeored by an agency of the United States Government. Neither the United States Government nor the University of California nor any of their employees, makes any warranty, express or implied, or acoumes any legal liability or reeponsibility for the accuracy, completeness, or usefulness of any information, apparatus, product, or procese disclosed, or represents that its use would not infringe privately owned rights. Reference herein to any upecific commercial products, process, or service by trade name, trademark, manufacturer, or otherwise, does not necesarily constitute or imply its endorsement, recommendation, or favoring by the United States Government or the University of California. The views and opinions of authors expressed herein do not necesearily otate or reflect those of the United States Government or the University of California, and shall not be used for advertioing or product endorsement purposes.

\author{
Printed in the United States of America \\ Available from \\ National Technical Information Service \\ U.S. Department of Commerce \\ 5285 Port Royal Raad \\ Springfield, VA 22161
}

Price

Code

A01

Papercopy Prices

A02

A03

A04

A05

A06

A07

A08

A09
Page

Range

Microfiche

CO1-050

051-100

101-200

201-300

301-400

401-500

501-600

601

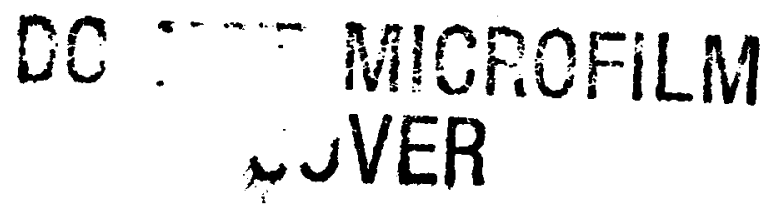




\section{DISCLAIMER}

This report was prepared as an account of work sponsored by an agency of the United States Government. Neither the United States Government nor any agency Thereof, nor any of their employees, makes any warranty, express or implied, or assumes any legal liability or responsibility for the accuracy, completeness, or usefulness of any information, apparatus, product, or process disclosed, or represents that its use would not infringe privately owned rights. Reference herein to any specific commercial product, process, or service by trade name, trademark, manufacturer, or otherwise does not necessarily constitute or imply its endorsement, recommendation, or favoring by the United States Government or any agency thereof. The views and opinions of authors expressed herein do not necessarily state or reflect those of the United States Government or any agency thereof. 


\section{DISCLAIMER}

Portions of this document may be illegible in electronic image products. Images are produced from the best available original document. 




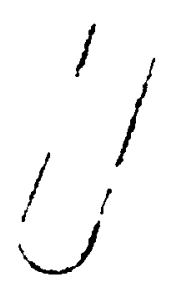

UCID--20995

DE87 006887

EXTENDED ANALYSIS OF CONSTANT HEIGHT HYDRAULIC FRACTURES

FOR THE ESTIMATION OF IN-SITU CRACK-OPENING MODULUS

FROM BOTTOMHOLE PRESSURE RECORDS.

By

Ananda M. Wi jesinghe

Earth Sciences Department

Lawrence Livermore National Laboratory

November, 1986.

\section{ABSTRACT}

Hydraulic fractures created in oil and gas bearing rock formations can be made to propagate for a limited time at approximately constant height if favorable stress, deformation modulus or fracture toughness barriers to height growth exist and if the fracture design is suitably optimized to exploit these favorable conditions and reduce height growth.

In this report, a unified theoretical formulation for the Perkins-Kern-Nordgren (PKN) and Christianovitch-Geertsma-De Klerk-Daneshy (CGDD) constant height fracture models is first presented. For a fracture fluid injection rate that varies as an arbitrary power of time, growth laws for fracturing fluid pressure, fracture width, and flow rate are rigorously derived for PKN and CGDD types of fractures. These similarity solutions account for non-Newtonian power-law fluid flow, transient fluid storage and generalized power-law fluid leak-off to the rock formation. They include and extend the results currently available in the literature for PKN and CGDD fractures.

The results for PKN and CGDD fractures are then generalized to obtain an approximate hybrid CGDD-PKN fracture model that can be applied to constant height fractures of arbitrary length/height aspect ratio and

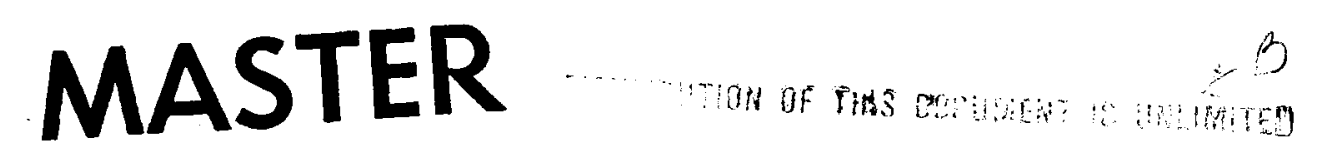


arbitrary cross-sectional shape. Characteristic times for fracture extension are identified and estimates are given for the transition times when the fracture evolves from a CGDD-type fracture at small aspect ratio to a PKN-type fracture at large aspect ratio. These results are useful for interpreting fracturing data and for designing fractures for crack-opening modulus measurements.

Because a hydraulic fracture responds to the in-situ properties of a large volume of rock, at least as large as itself, fracturing can be an attractive means of determining the in-situ deformation moduli of a rock mass at different length scales. With this motivation, for fracturing conditions that lead to constant height fracture extension, a procedure based on the hybrid CGOD-PKN model is proposed for computing the crack-opening modulus from suitable bottomhole pressure data. Sensitivity analysis of the uncertainty in the measured crack-opening modulus indicates that the crack-opening modulus computed from the initial CGDD-type fracture extension phase (if present) will be much less sensitive to the uncertainty in fracture-height than that computed from the final PKN-type fracture extension phase. If the crack-opening modulus is to be computed from the final PKN-type fracture extension period, the fracture height must be known with much less uncertainty than the uncertainty in the crack-opening modulus that is to be resolved through such measurements.

\section{DISCLAIMER}

\footnotetext{
This report was prepared as an account of work sponsored

Government. Neither the United States Government nor any an agency of the United States bility for the accuracy, completexpress or implied, or assumen agency thereof, nor any of their process disclosed, or represents that, or usefulness of any informatiogal liability or responsience herein to any specific commerts use would not inf ing manufacturer, or otherwise does not product, process, or service by owned rights. Refermendation, or favoring by the United necessarily constitute or ice by trade name, trademark and opinions of authors expressed States Government or imply its endorsement, recomUnited States Government or any agenein do not necessarily any agency thereof. The views
} 


\section{CONTENTS}

1. INTRODUCTION. ............................ 1

1.1 Background And Motivation.................... 1

1.2 Existing Hydraulic Fracturing Models............... 3

1.3 Scope of The Present Work................... 5

2. MODEL FORMULATION FOR CONSTANT HEIGHT FRACTURES.......... 9

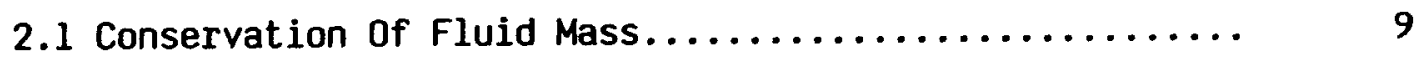

2.2 Flow Law For Non-Newtonian Fracturing Fluids......... 11

2.3 Crack-Opening Relation...................... 13

2.3.1 PKN Crack-Opening Model................. 13

2.3.2 CGDD Crack-Opening Model................ 13

2.4 Fluid Leak-0ff Model...................... 15

2.5 Governing Partial Differential Equation........... 16

3. SIMILARITY SOLUTIONS FOR PKN AND CGDD MODELS........... 18

3.1 General Analysis of Constant Height Fractures......... 18

3.2 Initial And Boundary Conditions For Dimensionless

Governing Equations........................ 25

3.3 Model Specific Analyses of Constant Height Fractures

For PKN And CGOD Fractures................... 26

3.3 .1 Power-Law Exponents.................... 27

General Case.......................... 27

Case of Small fluid Leak-0ff............... 29

Case of Small fluid Storage................ 29

3.3.2 Power-Law Coefficients................. 30

General Case........................ 31

Case of Small Fluid Leak-0ff.............. 32

Case of Small fluid Storage............... 32

3.3.3 Values of Dimensionless Parameters $\alpha$ And B.... 34

Case of Small Fluid Leak-0ff............... 34

Case of Small fluid Storage............... 35 
CONTENTS (continued)

Page

4. EXTENDED ANALYSIS FOR FRACTURES WITH ARBITRARY ASPECT

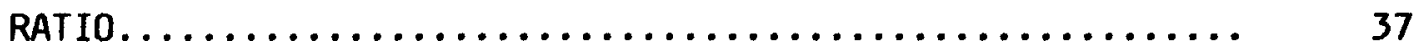

4.1 The Hybrid CGDD-PKN Fracture Model.............. 37

4.1.1 Characteristic Times For Fracture Extension For

PKN And CGDD Fractures................. 37

Case of Small Fluid Leak-Off............... 39

Case of Small Fluid Storage............... 39

4.1.2 Aspect Ratio Limiting Times For

PKN And CGOD Fractures.................. 39

Case of Small Fluid Leak-0ff.............. 40

Case of Small Fluid Storage................ 40

4.2 Bottomhole Pressure Response of The Hybrid CGDD-PKN

Fracture Model............................ 41

5. SENSITIVITY ANALYSIS AND ERROR ESTIMATES ............ 46

5.1 Simplifying Assumptions.................... 46

5.2 Bottomhole Pressure Time Response of PKN And CGDD

Fractures............................ 47

5.3 Sensitivity of The Bottomhole Pressure Coefficient

To Crack-Opening Modulus And Fracture Height.......... 49

5.3 .1 Case of Small Fluid Leak-off............... 50

5.3 .2 Case of Small fluid Storage............... 50

5.4 Uncertainty In The Calculated Crack-Opening Modulus

Due To Uncertainty In Fracture Height............. 51

5.4 .1 Case of Small Fluid Leak-0ff............... 54

5.4 .2 Case of Small Fluid Storage................ 54

6. SUMMARY AND CONCLUSIONS...................... 58

6.1 Modelling Results......................... 58

6.2 Estimation of Crack-Opening Modulus.............. 60 


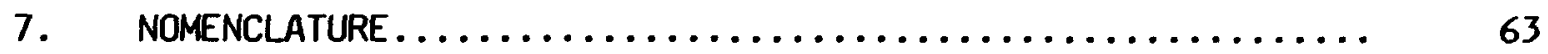

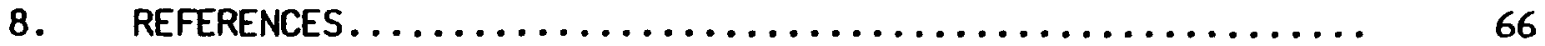

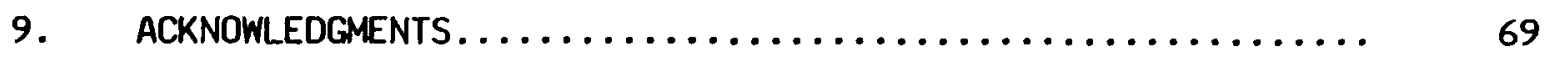




\section{INTRODUCTION}

\subsection{Background And Motivation}

Hydraulic fracturing is a process by which fractures are created in underground rock formations by injecting fluid through a wellbore at a pressure high enough to fracture the rock. Hydraulic fracturing has excited scientific interest because of the fascinating interactions between the physical processes that govern it and the challenge posed by the difficulties of its analysis. In the scientific arena it has found application in determining the state of stress in the earth's crust $[1,11,14]$, in explaining the fracturing phenomena that occur naturally in the subsurface $[5,29]$ or in man-made structures such as dams and tunnels, and in studies of the escape of gaseous contaminants from underground explosions $[15,32]$. Perhaps of greater import is its economic impact on the cost effective extraction of underground petroleum and geothermal energy resources and on the subsurface disposal of liquid wastes. For example, during the last forty years, hydraulic fracturing has been used to increase production from wells drilled in oil and gas reservoirs $[13,35]$ and in potable aquifers [33], to enhance the injectivity of liquid waste disposal wells [36], to create fractured geothermal energy reservoirs in hot dry rock masses [25] and to measure the in-situ stresses in reservoir rock formations for fracture design purposes $[1,35]$.

The propagation of hydraulically induced fractures in the subsurface is influenced by in-situ conditions such as the state of stress in the targeted and neighboring rock formations, the spatial distribution of deformation moduli and fracture toughness of the rock, and the permeability, fluid saturation and pore pressure levels that affect fluid-loss to the porous rock formation. In addition, the fluid injection schedule, the rheology and fluid-loss control properties of the fracturing fluid, and the properties and schedule of the proppant are control parameters that affect the fracturing process. Many properties, such as the fluid rheology parameters and the fluid-loss coefficients that are required as input data to fracture design models, are measured in the laboratory. We wish to focus attention here on the crack-opening modulus $E_{c}=G /(1-v)$, where $G=$ shear 
modulus and $v=$ Poisson's ratio, that is the only deformation modulus used in most simple fracture design models.

There is increasing evidence that suggests that static deformation moduli measured in the laboratory and dynamic deformation moduli measured by sonic well-logging methods in the field may not be representative of the deformation modulus experienced in-situ by a propagating fracture. Because of the quasi-static nature of hydraulic fracturing and the larger levels of strain induced by hydrofractures, it would seem from a conceptual point of view that the static moduli, and not the dynamic moduli, are the deformation properties that should be used for designing hydraulic fractures. There is a large collection of data $[17,18,19,20]$ that show that static deformation moduli measured in the laboratory differ significantly (60\% to 600\%) from the dynamic moduli measured both in the laboratory and in the field on the same rock under equivalent stress conditions. There are even substantial differences between the sonic modulus measurements made in the laboratory and in the field.

Perhaps of greater relevance to fracture design is the possibility that in-situ static moduli can be very different from those measured in the laboratory due to the scale dependence of the moduli that are to be measured. Spatial scale dependence of deformability has been documented in the geotechnical engineering literature [12] and arises as a result of natural fractures and modulus inhomogeneities that occur in the field on scales much larger than the sizes of laboratory rock samples. The larger length scales associated with these features are likely to be more representative of the length scales affecting the rock mass stiffness experienced by a large hydraulic fracture. It is implied in these statements that the rock mass, though discontinuous and inhomogeneous, can be viewed as a continuum on the spatial scales on which we wish to assign average modulus values. If the distribution of these features is too sparse to permit such an averaging procedure, then the measured moduli cannot be considered to be constitutive properties but merely deformability measures that depend upon the specific test configuration and interpretive model used. While the observed differences between the moduli measured by different methods may in part be related to factors other than scale 
dependence, such as sample disturbance during removal or undetected local conditions occurring within or near the wellbore in the field measurements, it is imperative to try to develop an independent method of determining the in-situ stiffness properties of the rock mass on the length scale of the hydraulic fractures.

From its very early days $[14,11,1]$ the potential of hydraulic fracturing for acquiring in-situ stress data was recognized; now this is often a routine preliminary procedure in the design of massive hydraulic fractures [35]. However, the possibility of using hydraulic fracturing for determining in-situ deformation moduli does not seem to have been explored. We propose here to back-calculate the crack-opening modulus from hydraulic fracturing bottomhole pressure data using an interpretive model that is appropriate for the conditions in the field. Because a hydraulic fracture responds to the in-situ properties of a large volume of rock, at least as large as itself, fracturing can be an attractive means of assessing the scale-dependence of deformation moduli of the rock mass in-situ. The idea is even more attractive when it is realized that the fracture dimensions can be controlled by controlling the pumping schedule and the fracturing fluid properties so that, conceptually, it should be possible to sample the stiffness properties of the rock formation at different length scales. A major difficulty, of course, is the possibility that the discontinuities or inhomogeneities are sparsely distributed and influence fracture propagation only as discrete entities. Another difficulty that can arise is that, although the discontinuities and inhomogeneities are sufficiently profuse to be viewed as continuum features, the fracture may intercept one or more of them leading to a situation for which the data required for interpretation cannot be easily acquired in sufficient detail even if a capability to analyze the problem could be developed.

Subject to these caveats on the fidelity and sophistication of our models and the availability of supporting data, it may be possible to calculate the modulus on different length scales using data from a single fracture as it extends with time. An alternative procedure is to assign a single different modulus to each fracture of a different size. For this to be possible, a reliable means of analyzing hydraulic fracturing pressure 
records is an essential prerequisite. The suitability of existing hydraulic fracturing models must, therefore, be examined and methods of interpretation and, if necessary, improved models must be developed for this purpose. In addition, the sensitivity of the calculated moduli to the analysis procedure and the physical parameters governing the hydraulic fracturing process must be evaluated. The results presented in this report constitute a first step in this direction.

\subsection{Existing Hydraulic Fracturing Models}

Interpretive models of hydraulic fracturing that have been reported in the open literature over the years can be conveniently classified into first, second and third generation models. An exhaustive inventory of these models is not attempted here. Instead, we refer interested readers to the review by Mendelsohn [22,23] and cite only a few models that are either representative of a class or are distinctly different in approach. The first generation models are those which assume that the height of the fracture is constant in time and uniform in space, and that the resistance of the fracture to opening under fluid pressure is derived from either the lateral or the longitudinal direction of the fracture. The best known of these are the Perkins-Kern-Nordgren (PKN) and Christianovitch-Geertsma-De Klerk-Daneshy (CGDD) models $[31,28 ; 16,37,9,7]$. They incorporate varying degrees of approximation for the crack-opening stiffness, the conservation of fluid mass in the fracture, the flow law relating the flow rate to the pressure gradient, and the fluid leak-off into the porous rock formation. Few, if any, of the first generation models include all of these effects with a sufficient degree of generality [10]; this provides the motivation for the derivation of the extended results for constant height fractures and the hybrid model described in the present report.

The second generation models differ from those of the first generation in that they allow simultaneous growth of both fracture height and fracture length. The pseudo-three-dimensional P3DH-type models, first introduced by cleary [6], allow for height growth by coupling CGDD-type fracture extension in the lateral direction with PKN-type fracture extension in the longitudinal direction. The coupling is through the equation of fluid mass 
conservation. This approach yields useful results but requires a great deal of subjective judgement in choosing model-dependent parameters. Other, distinctly different, approximate models that allow simultaneous height and length growth have been introduced by Stout [34] and by Advani et. al. [3]. Both of these models assume a particular shape for the fracture geometry that is parameterized through time dependent dimensions. The ordinary nonlinear differential equations that govern the growth of these dimensions in time are obtained by Advani et. al. using a Lagrangian formulation with non-conservative generalized forces and by Stout by means of a full-fledged rate variational principle. Advani et. al. obtained approximate power-law solutions by resorting to approximations regarding the stiffness contributions from the two principal directions of the assumed fracture shape, while Stout integrated his ordinary differential equations by an explicit numerical integration procedure.

Third generation models allow for the evolution of an arbitrary fracture shape but still confine the fracture to growth in a single vertical plane. These models are based on either hybrid boundary element-finite element formulations such as that of Abou-Sayed et. al. [2] requiring discretization of only the fracture surface or full-fledged finite-element simulators such as that reported recently by Morita et. al.[24]. These models require substantially greater computational effort but provide more flexibility in modelling the actual field conditions. Used with care, they have the potential to provide the most accurate predictions and can be used to calibrate and define the domains of applicability of simpler, less accurate but more convenient, models.

\subsection{Scope of The Present Work}

The present study is restricted to first generation models which assume that the hydraulic fracture remains confined to a single vertical plane passing through the wellbore and that it extends laterally (i.e. horizontally) away from the wellbore at constant fracture height. It is assumed that the orientation of the fracture is determined by the direction of the minimum in-situ principal stress in the far-field and that the minimum principal stress acts in the horizontal plane. Therefore, the plane of the 
fracture is vertical and its azimuthal orientation is normal to the minimum in-situ principal stress. In a practical sense, this implies that such fractures will be created only at depths sufficiently below the surface where the vertical stress will not be the minimum in-situ principal stress. It also implies that the difference between the minimum and intermediate principal stresses is sufficiently large to fix and contain the fracture within a single unique vertical plane as it propagates in the horizontal direction. The PKN and CGDD models, the best known models of this class, are distinguished primarily by whether the stiffness contribution to opening of the fracture by the fracturing fluid pressure is derived from the lateral (i.e. vertical) direction or the longitudinal (i.e. horizontal) direction of the fracture.

In the PKN model, shown in Figure 1 , it is assumed that the fracture closes at the top and the bottom of the fracture in the vertical direction and that the resistance of the fracture to opening under fluid pressure is derived solely from the elastic stiffness of its cross-section in the vertical direction. This implies that the vertical cross-sections of the fracture deform independently of each other and, therefore, any contribution to the stiffness from the horizontal direction is neglected. On the other hand, in the CGDD model, also shown in Figure 1 , it is assumed that the cross-sections are of uniform width and do not close at the top and bottom so that the resistance of the fracture to opening under fluid pressure is derived solely from its elastic stiffness in the horizontal direction. The uniform width assumption would be strictly valid, for example, if the fracture terminates in the vertical direction at rock boundaries at which there is complete slip. In both models the fluid flow in the fracture is assumed to be pseudo-steady and to take place horizontally while the fracture extends in this direction. Within the confines of these major distinguishing assumptions, power-law fluid flow, proppant transport and approximate one-dimensional fluid leak-off into the porous rock formation have been incorporated in these two models.

In this report a unified theoretical formulation for constant height fractures of the PKN and CGDD types is presented in Section 2. The governing equations for constant height fractures of arbitrary cross-sectional 


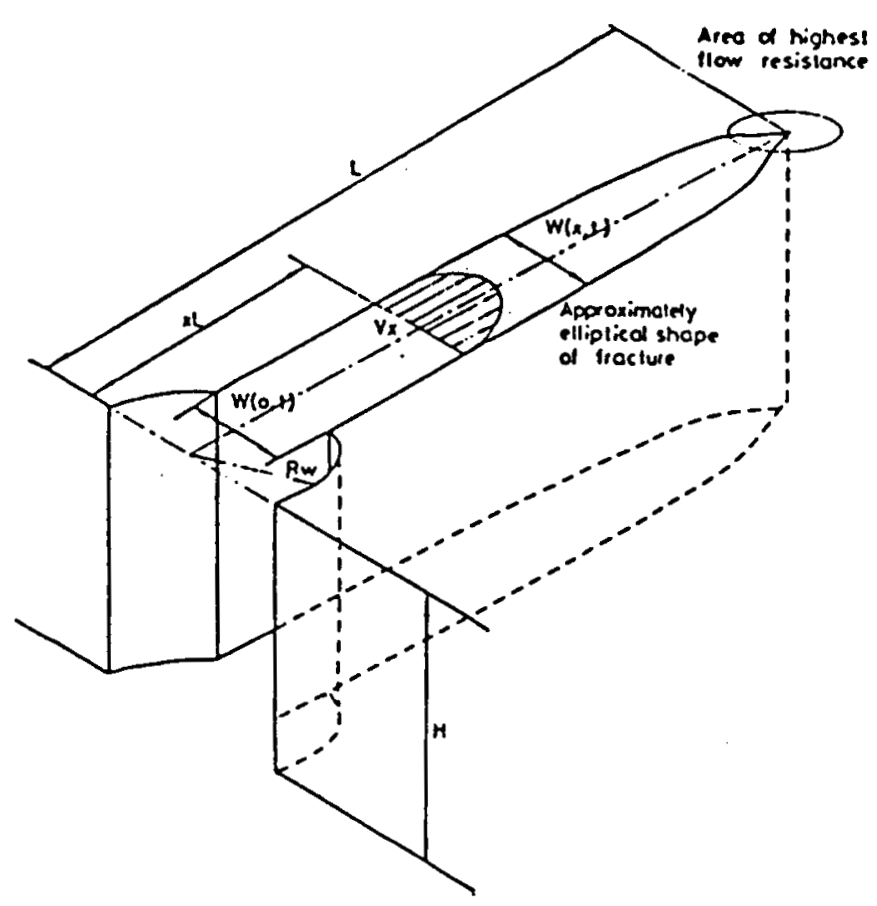

CGDD FRACTURE MODEL

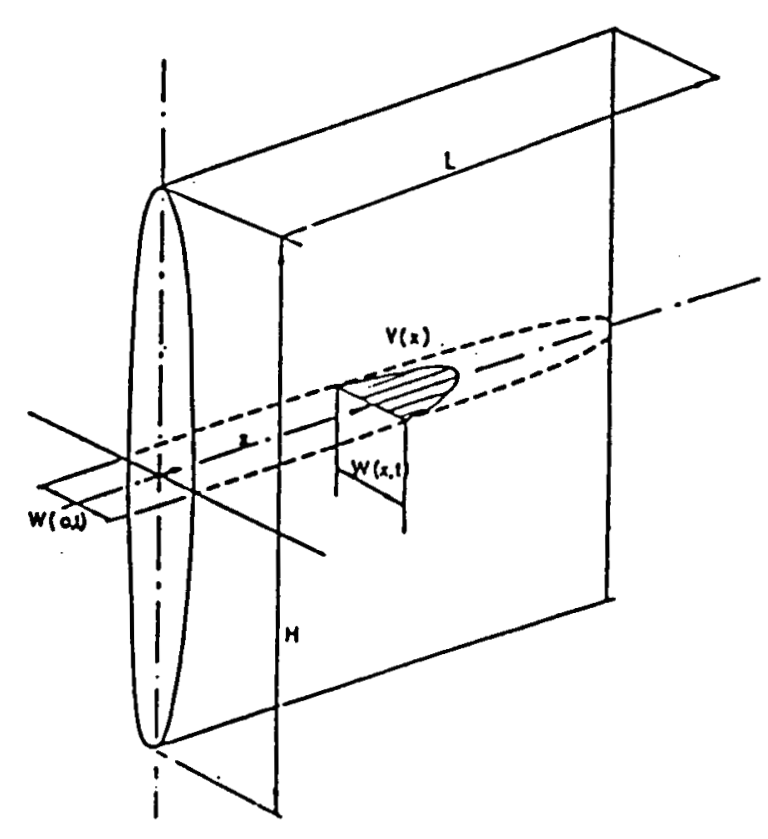

PKN FRACTURE MODEL

FIGURE 1: CONSTANT HEIGHT FRACTURE MODELS BY PERKINS-KERNS-NORDGREN (PKN) AND CHRISTIANOVICH-GEERTSMA-DE KLERK-DANESHY (CGDD). 
shape with non-Newtonian power-law fluid flow in the fracture and a generalized power-law fluid leak-off model are given here. In Section 3 the equations are recast in dimensionless form and similarity solutions for fluid pressure, fracture width, flow rate and fracture length that grow as powers of time are derived. The resulting nonlinear ordinary differential equations are not completely solved but their limiting behaviors are investigated. Although the distributions of the variables over the fracture are not determined, the similarity analysis enables the dependence on time, and on the physical properties that govern the fracturing process, to be extracted. The time regimes within which either the fluid storage term or the fluid leak-off term can be neglected are identified in terms of times that are characteristic of PKN and CGDD types of fracture extension.

In Section 4 these results are extended to a fracture whose cross-section always remains closed at its top and bottom edges but which extends at constant height. It is shown that such a fracture can be considered to be a hybrid CGDD-PKN fracture which behaves as a CGOD fracture when its length is much smaller than its height but which evolves into a PKN fracture when the fracture length is much greater than the fracture height. Estimates of the times of transition between these two behaviors are derived as an important by-product of this extended view. In Section 5 the results derived for the power-law coefficients and exponents are used to devise a procedure for computing the crack-opening modulus from bottomhole pressure records obtained for a hybrid CGDD-PKN constant height fracture. A sensitivity analysis of the impact of uncertainty in the assumed fracture height on the crack-opening modulus is presented next. The conclusions based on the extended analysis of constant height fracture models and the sensitivity analysis for the crack-opening modulus are summarized in Section 5. 


\section{MODEL FORMULATION FOR CONSTANT HEIGHT FRACTURES}

The governing equations are derived here for PKN and CGDD-type hydraulic fractures of uniform and constant height propagating in a homogeneous fluid saturated porous rock formation. Consider the flow of the injected fracturing fluid along a uniform plane fracture of height $H$ and an as yet unspecified cross-sectional shape, as shown in Figure 1 . We assume that the plane of the fracture is oriented normal to the minimum in-situ principal stress $S$ acting in the far-field and that the in-situ stress $S$ and pore fluid pressure $p_{i}$ distributions are uniform in the far-field. Injection of fracturing fluid is begun at time $t=0$ at a rate $Q_{w}(t)$ and the bottomhole fluid injection pressure $P_{w}(t)$ required to maintain this injection rate is measured. The present analysis attempts to approximately predict the bottomhole injection pressure $p_{w}(t)$, the fracture length $L(t)$

and the crack-opening width $w(x, z, t)$ of the fracture as a function of the in-situ stress, the mechanical properties of the rock formation and the rheological properties of the fracturing fluid. In particular, we wish to assess the sensitivity of the bottomhole pressure history to the mechanical properties of the rock formation and the extent of the uncertainty in the prediction of these properties from bottomhole pressure data that results from the approximations embedded in the models.

\subsection{Conservation of Fluid Mass}

For one-dimensional fluid flow along the length of the fracture, conservation of fluid mass in the fracture is approximately expressed in terms of average cross-sectional quantities by Equation (2.1):

$$
\frac{\partial\left(\rho W H Y_{W}\right)}{\partial t}+\frac{\partial(\rho Q)}{\partial x}+2 \rho Y_{L} H q_{L}=0 \text {. }
$$

In Equation (2.1), $Y W(x, t)$ and $\rho(x, t)$ are the average fracture width and the average fluid density, respectively, $Q(x, t)$ is the total fluid flow rate along the fracture and $q_{L}(x, t)$ is the total rate of fluid loss to the porous formation per unit fracture area at any cross-section of the 
fracture. To account for only a part of the height of fracture being adjacent to a permeable rock formation, the fluid leak-off rate $q_{L}$ is defined with respect to a leak-off fracture height $H_{L}=\gamma_{L} H$ that is less than the height of the fracture. The total flow rate $Q(x, t)$ is equal to the integral over the height of the fracture of the local flow rate $q_{x}(x, z, t)$ in the longitudinal direction. The local fluid velocity has components $v_{x}, v_{y}$ and $v_{z}$ in the longitudinal, lateral and normal directions with respect to the fracture surface. The local flow rate $q_{x}(x, z, t)$ is, therefore, equal to the flow velocity $v_{x}(x, y, z, t)$ at a fixed height $z$ integrated with respect to $y$ over the fracture width $w(x, z, t)$. These quantities are defined by:

$$
\begin{aligned}
Y_{W} W(x, t) & =\frac{1}{H} \int_{-H / 2}^{+H / 2} W(x, z, t) d z, \\
\rho(x, t) & =\frac{1}{H} \int_{-H / 2}^{+H / 2} d(x, z, t) d z, \\
Q(x, t) & =\int_{-H / 2}^{+H / 2} q_{x}(x, z, t) d z, \\
q_{L}(x, t) & =\frac{1}{H_{L}} \int_{-H_{L} / 2}^{+H_{L} / 2}\left\{V_{y}(x, w / 2, z, t)-V_{y}(x,-w / 2, z, t)\right\} d z,
\end{aligned}
$$

where, $\gamma_{W}$ is a shape factor that depends on the assumed shape of the cross-section of the fracture and $d(x, z, t)$ is the average fluid density over the width of the fracture. If the product form given by Equation (2.6) is assumed for the cross-sectional shape, then the shape factor $\gamma_{W}$ is defined by Equation (2.7). For PKN fractures with elliptical cross-sections and CGDD fractures with uniform cross-sections, the cross-sectional shape function $f_{W}(\zeta)$ (assumed to be an even function of $\zeta=2 z / H$ ) and $\gamma_{W}$ are given by Equations (2.8) and (2.9), respectively.

$$
\begin{aligned}
& w(x, z, t)=W(x, t) f_{W}(2 z / H) . \\
& Y_{W}=\frac{1}{H} \int_{-H / 2}^{+H / 2} f_{W}(2 z / H) d z .
\end{aligned}
$$


Elliptic PKN cross-sections: $f_{W}(\zeta)=r\left(1-\zeta^{2}\right) ; \gamma_{W}=\pi / 4$.

Constant width CGOD cross-sections: $f_{W}(\zeta)=1 ; \gamma_{W}=1$.

If the fluid compressibility is small compared to the compliance of the fracture width under the normal effective stress $(p-S)$ (an assumption valid for liquid saturated fractures), then Equation (2.1) simplifies to Equation (2.10) for constant height fractures:

$$
\gamma_{W} H \frac{\partial W}{\partial t}+\frac{\partial Q}{\partial x}+2 \gamma_{L} H q_{L}=0 \text {. }
$$

\subsection{Flow Law For Non-Newtonian Fracturing Fluids}

For low Reynold's Number of the fluid flow in the fracture, the pressure gradient in the flow direction is balanced primarily by the viscous shear forces as given by Equation (2.11). The rheological behavior of proppant laden fluid is further complicated by the presence of fluid loss control and viscosity enhancing chemical additives. We conform to standard practice here and approximate the non-Newtonian rheology of this composite fluid-solid mixture by the power-law relationship given by Equation (2.12), where $K^{\prime}$ ' is the fluid consistency and $n^{\prime}$ is the power-law exponent. The power-law exponent ranges between 0 and 1 , and when $n^{\prime}=1$ the fluid is Newtonian and the fluid consistency $K^{\prime}$ ' is the Newtonian fluid viscosity. When Equation (2.12) is used to eliminate $\tau_{x y}$ from Equation (2.11), Equation (2.13) that governs power-law fluid flow in the fracture is obtained.

$$
\begin{gathered}
\frac{\partial p}{\partial x}=\frac{\partial \tau_{x y}}{\partial y}, \\
\tau_{x y}=-K^{\prime}\left|\frac{\partial v_{x}}{\partial y}\right|^{\left(n^{\prime}-1\right)} \frac{\partial v_{x}}{\partial y}, \\
\frac{\partial p}{\partial x}+\frac{\partial}{\partial y}\left\{K^{\prime}\left|\frac{\partial v_{x}}{\partial y}\right|^{\left(n^{\prime}-1\right)} \frac{\partial v_{x}}{\partial y}\right\}=0 .
\end{gathered}
$$


In writing Equation (2.13) we have neglected the unsteadiness of the fluid flow, the convective inertial effects and the effects of fluid leak-off on the local velocity profile. This Equation can be easily solved for the fluid velocity $v_{x}$ subject to no-slip boundary conditions on the fracture surfaces, to arrive at the following relationships for the velocity profile (Equation (2.14)) and the local flow rate $q_{x}(x, z, t)$ (Equation (2.15)) as a function of the pressure gradient:

$$
\begin{aligned}
& v_{x}=\left(\frac{n^{\prime}}{n^{\prime}+1}\right)(w / 2)^{\left(n^{\prime}+1\right) / n^{\prime}}\left(-\frac{1}{K^{\prime}} \frac{\partial p}{\partial x}\right)^{1 / n^{\prime}}\left\{1-|2 y / w|^{\left(n^{\prime}+1\right) / n^{\prime}}\right\}, \\
& q_{x}=\left(\frac{2 n^{\prime}}{2 n^{\prime}+1}\right) H(w / 2)^{\left(2 n^{\prime}+1\right) / n^{\prime}}\left(-\frac{1}{K^{\prime}} \frac{\partial p}{\partial x}\right)^{1 / n^{\prime}} .
\end{aligned}
$$

Equation (2.15) can now be substituted in Equation (2.4) to obtain the desired flow law between the total flow rate $Q(x, t)$ and the pressure gradient:

$$
\left(\frac{6 Q}{H}\right)=\left(-\frac{\gamma_{Q} W^{\left(2 n^{\prime}+1\right)}}{2 K_{a}} \frac{\partial p}{\partial x}\right)^{1 / n^{\prime}} .
$$

The apparent fluid viscosity $K_{a}$ and the shape parameter $\gamma_{Q}$ in Equation (2.16) are defined by:

$$
\begin{aligned}
K_{a} & =k^{\prime}\left(\frac{2 n^{\prime}+1}{3 n^{\prime}}\right)^{n^{\prime}}, \\
r_{Q} & =\left\{\int_{0}^{1} f_{W^{(\zeta)^{2}}}^{\left.\left(2 n^{\prime}+1\right) / n^{\prime} d \zeta\right\}^{n^{\prime}},}\right. \\
& =\left\{\int_{0}^{1}\left(1-\zeta^{2}\right)^{\left(2 n^{\prime}+1\right) / 2 n^{\prime}} d \zeta\right\} n^{n^{\prime}} \text { for elliptic PKN cross-sections, } \\
& =1 \quad \text { for constant width CGDD cross-sections. }
\end{aligned}
$$

The shape parameter $\gamma_{Q}$ can be computed for any specified value of $n^{\prime} ;$ for $n^{\prime}=1$, for example, it takes the value of $Y_{Q}=3 \pi / 16$. Also, the standard Poiseuille flow law for Newtonian fluid flow between parallel plates can be recovered from Equation (2.16) for a constant width fracture by setting $n^{\prime}=1$. 


\subsection{Crack-Opening Relation}

The final relationship required to achieve closure of Equations (2.10) and (2.16) is that between the fracture width and the effective stress distribution $(p(x, t)-S)$ acting on the fracture surfaces. The principal difference between the PKN and CGOD models stems from the differences in this relationship in the two models.

\subsubsection{PKN Crack-Opening Model}

In the PKN model, vertical cross-sections of the fracture act independently of each other by assumption so that approximately plane strain conditions prevail at each cross-section. Therefore, for the elliptic cross-sectional shape, and the uniform fracture fluid pressure within the fracture acting at each cross-section, the fracture width $w(x, z, t)$ is given as a function of $(p(x, t)-s)$ by:

$$
\begin{aligned}
w(x, z, t) & =\frac{1}{E_{c}} H(p(x, t)-S) f_{W}(2 z / H), \\
W(x, t) & =\frac{1}{E_{c}} H(p(x, t)-S),
\end{aligned}
$$

where the crack-opening modulus $E_{c}=G /(1-v)$ and $f_{W}(2 z / H)$ is defined by Equation (2.8).

\subsubsection{CGDD Crack-Opening Model}

In the CGDD model, each cross-section is assumed to be of uniform width and the elastic stiffness of the fracture is, therefore, derived solely from its deformation in the lengthwise direction. The fluid pressure varies along the fracture due to the viscous shear of the fracturing fluid with the result that the fracture width at any location is related to the entire distribution of pressure over the fracture length. For the plane strain conditions prevailing in the horizontal plane, the elastic solution to the crack width for an arbitrary pressure distribution acting on its surface was given by England and Green [8] as: 


$$
W(n, t)=\frac{2 L(t)}{E_{c}}\left\{\frac{2}{\pi} \int_{n}^{1} \int_{0}^{\zeta} \frac{\zeta(p(\xi, t)-S)}{v\left(\zeta^{2}-n^{2}\right) v\left(\zeta^{2}-\xi^{2}\right)} d \xi d \zeta\right\},
$$

where $n=x / L(t)$.

In PKN fractures the cross-section was assumed to be of elliptical shape with fluid penetrating to the very edges of the fracture cross-section at uniform pressure. Under these conditions, linear elasticity theory predicts infinite stresses at the crack edge although the width profile is well defined. Because the fracture height was assumed to be constant, it was reasonable to ignore this situation in the case of PKN fractures. However, if this condition were to prevail in the case of CGDD fractures, infinite stresses would exist at the crack-tip that is responsible for fracture extension in the lengthwise direction. This dilemma was resolved by Khristianovitch and Zheltov [16,37] who suggested that the pressure distribution within the crack would adjust itself to yield a crack-width profile that not only would close at the crack-tip but also would close smoothly at the crack-tip. The condition of smooth closure of the fracture tip was later placed on a firmer fracture mechanics foundation by Barenblatt [4]. This inspired hypothesis is similar to the Kutta-Joukowsky-Chaplygin condition [21] for potential fluid flow past an aerofoil with a sharp trailing edge. In this fluid mechanics problem, the streamlines are required to be tangential to the aerofoil at the trailing edge to eliminate the infinite fluid velocities that would otherwise exist at this point. The Kutta-Joukowsky-Chaplygin condition determines the lift on the aerofoil while the Khristianovitch-Zheltov condition determines the length of the crack. In contrast to this situation for CGDD fractures, the governing equation for crack width for PKN fractures is of a lower order than the integro-differential equation for CGDD fractures and, therefore, can support only the condition that the crack closes at the crack-tip.

When the gradient $d W(n) / d n$ is set equal to zero in Equation (2.21) we obtain Equation (2.22a) as the condition that must be satisfied by the pressure distribution to achieve smooth closure at the crack-tip. When 
the finite tensile strength (or fracture toughness) of the rock is taken into account we obtain Equation (2.22b). If the fracture length is large enough to satisfy the Inequality in (2.22a), where $\sigma$ is the specific surface energy of the rock, the influence of fracture toughness of the brittle rock is small and can be neglected $[9,30]$ and Equation (2.22a) is recovered.

$$
\begin{aligned}
\int_{0}^{1} \frac{(p(\eta, t)-S)}{r(1-\eta)} d \eta & \simeq 0 \quad \text { for } L(t)>>\frac{2 k^{2}}{\pi^{2} s^{2}}, \\
& =\frac{k}{\sqrt{(2 L(t))}},
\end{aligned}
$$

with $K=\left(2 \pi E_{c} \sigma\right)^{1 / 2}$.

Geertsma and De Klerk [9] have shown that, except in a small narrow wedge-like zone near the tip of the fracture, for many hydraulic fracturing conditions the fracture width profile can be approximated as being elliptical in shape:

$$
W(x, t)=\frac{2}{E_{c}} L(t)(\bar{p}(t)-S) f_{W}\left(\frac{x}{L(t)}\right)
$$

where, $\bar{p}(t)$ is the average fluid pressure in the fracture and $f_{w}(n)$ is the shape function defined previously by Equation (8) for elliptic crosssections.

\subsection{The Fluid Leak-off Model}

The fluid loss to the rock formation is assumed to be governed by a generalized form of Carter's fluid leak-off model given by Equation (2.24a):

$$
\begin{aligned}
q_{L}(x, t) & =\frac{c}{(t-\tau(x))^{m}}, \\
x & =L(\tau) .
\end{aligned}
$$


In this model, an arbitrary fluid leak-off power index $m$ is used instead of the standard value of $1 / 2$ because this value is only appropriate for leak-off controlled by linear pressure diffusion in the rock formation. As indicated by Equation (2.24b), $\tau(x)$ is the time at which the propagating $t$ ip of the fracture arrives at a location $x$ in the formation so that $(t-\tau(x))$ is the elapsed time during which fluid leaked-off into the rock formation since its first exposure to the fracturing fluid. In hydraulic fracturing numerical codes the leak-off coefficient $C$ is usually updated in time to reflect its dependence on the variation in the fluid pressure in the fracture. In the present analysis, however, we will assume $C$ to be a constant.

\subsection{Governing Partial Differential Equation}

With these definitions, the partial differential equation which governs the extension of both PKN and CGDD type fractures can be written by combining Equations (2.10), (2.16) and (2.24):

$$
\left.\frac{\partial W}{\partial t}+\frac{\partial}{\partial x}\left[{\frac{Y_{Q}}{6 \gamma_{W}}}_{-\frac{W}{2 K}^{\left(2 n^{\prime}+1\right)}} \frac{\partial p}{\partial x}\right\}^{1 / n^{\prime}}\right]+\frac{2 Y_{L}}{Y_{W}} \frac{c}{(t-\tau)^{m}}=0 .
$$

The values of the shape factors $\gamma_{W}$ and $\gamma_{Q}$ that appear in Equation (2.25) were given previously for both types of fractures. For PKN fractures, the crack-opening relation given by Equation (2.20) can be substituted in Equation (2.25) to obtain the governing Equation (2.26) solely in terms of the fracture width $w(x, t)$ :

$$
\frac{\partial W}{\partial t}+\frac{\partial}{\partial x}\left[\frac{1}{6 \gamma_{W}}\left\{-\frac{Y_{Q} E_{c} W^{\left(2 n^{\prime}+1\right)}}{2 H K_{a}} \frac{\partial W}{\partial x}\right\}^{1 / n^{\prime}}\right]+\frac{2 \gamma_{L}}{Y_{W}} \frac{C}{(t-\tau)^{m}}=0,
$$

while the bottomhole pressure $p_{w}(t)$ can be recovered from the fracture width at the wellbore from:

$$
p_{w}(t)=S+\frac{E}{H} w(0, t)
$$


In the case of CGDD fractures the situation is more complicated because $W(x, t)$ is related to the distribution of $p(x, t)$ over the entire fracture by Equation (2.21), or more approximately, through Equation (2.23) to the average pressure in the fracture $p(t)$. 


\section{SIMILARITY SOLUTIONS FOR PKN AND CGDD FRACTURES}

\subsection{General Analysis of Constant Height Fractures}

The standard method of solving the problem posed by Equations (2.7)-(2.10) would be to discretize them in space and/or time by a finitedifference or finite-element technique and to solve the resulting set of coupled nonlinear algebraic equations by some iterative scheme. For our present purpose, we are primarily interested in analyzing the time history of the bottomhole pressure for different pumping schedules at the wellbore and in evaluating its sensitivity to the parameters of the hydraulic fracturing process. With this limited aim, a similarity solution technique is devised here to extract the functional dependence of the bottomhole pressure on time and on the process parameters, for a limited but realistic class of power-law pumping schedules, without having to resort to the rigors of obtaining a complete numerical solution to the problem.

In doing so, we present a unified treatment of both PKN and CGDD fractures and extend the existing solutions to include fluid rheology, leak-off and pumping rate governed by generalized power-laws. The complete solution to the similarity solutions for PKN fractures was given by Nordgren [28] for the special case of a Newtonian fluid, the half-power fluid leak-off law and constant fluid injection rate. Cleary [6] has presented more general similarity solutions for PKN fractures with non-Newtonian power-law fluid rheology, generalized power-law fluid leak-off and power-law fluid injection rates. However, no computational results comparable to Nordgren's work were presented by him. Geertsma and De Klerk [9] provided an approximate solution for CGOD fractures that neglects the fluid storage term and approximates the crack-opening relationship. Their work is limited to Newtonian fluid flow in the fracture, half-power fluid leak-off to the rock formation and a constant rate of fluid injection. Daneshy [7] has reported an approximate extension of this model to power-law fracturing fluids but information is not available in sufficient detail regarding this improvement. In summary, a general theory has been presented by cleary for PKN fractures but no rigorous computations of the similarity equations are available. For CGDD 
fractures, no similarity analysis that accounts for all of the features we propose to include in the present model has been reported previously in the open literature. Furthermore, the results presented here on the characteristic times and aspect ratio limiting times for hybrid CGDD-PKN fractures are not available in the literature.

The solutions are found by first transforming the nonlinear partial differential Equation (2.10) in the two independent variables $x$ and $t$ to an ordinary differential equation in the single similarity variable $n$ defined by Equation (3.1). The similarity variable $\eta$ defined by this Equation is the dimensionless distance along the fracture and is equal to the actual distance scaled to the length of the fracture $L(t)$. We further assume that the fracture length $L(t)$ varies according to the power-law given by Equation (3.2). We seek similarity solutions for $p(x, t), W(x, t)$, $Q(x, t), q_{L}(x, t)$ in the form of Equations (3.3), (3.4), (3.5) and (3.6), respectively, where the corresponding power-law exponents $p, \omega, q, r$ and the dimensional coefficients $P_{0}, W_{0}, Q_{0}$ and $q_{L o}$ will be chosen to obtain a meaningfully scaled dimensionless governing equation that depends only on the similarity variable $\eta$ as the sole independent variable. The dimensionless pressure $p_{D}(n)$, the dimensionless fracture width $W_{D}(n)$, the dimensionless flowrate $Q_{D}(n)$ and the dimensionless leak-off rate $q_{L D}(n)$ provide convenient invariant means of representing the corresponding spatial distributions over the length of the fracture as it evolves in time.

$$
\begin{aligned}
n(x, t) & =x / L(t), \\
L(t) & =L_{0} t^{l}, \\
p(x, t)-S & =p_{0} p_{D}(n) t^{p}, \\
W(x, t) & =W_{0} W_{D}(n) t^{W}, \\
Q(x, t) & =Q_{0} Q_{D}(n) t^{q}, \\
q_{L}(x, t) & =q_{L 0} q_{L D}(n) t^{r} .
\end{aligned}
$$


As the first step we restrict the fracture fluid injection rate schedules to power-law pumping schedules of the form:

$$
Q_{w}(t) \quad=Q(0, t)=Q_{0} t^{Q},
$$

where $Q_{0}$ and $q$ are known input parameters that are consistent with the similarity assumption of Equation (3.5). Therefore, from Equation (3.5) we obtain the boundary condition:

$$
Q_{D}(0) \quad=1
$$

Note that for constant injection rate (i.e., $q=0$ ) $Q_{0}$ is the rate of injection itself but for $q>0$ it has physical units different from that of flow rate. To obtain the dimensionless form of the flow-law given by Equation (3.10) we use Equations (3.1-3.5) to substitute for the dimensional variables in Equation (2.16) and obtain Equation (3.9):

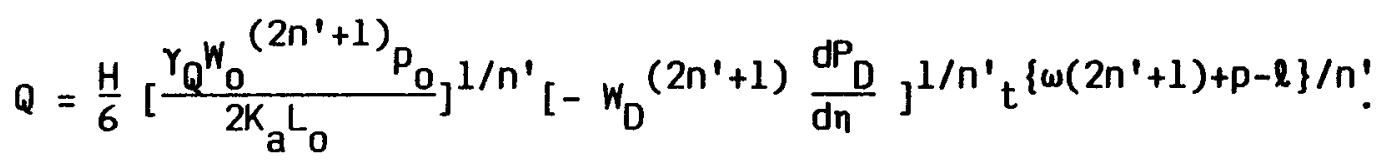

For this relation to be consistent with Equation (3.5) the power-law indices must be related by:

$$
\left\{w\left(2 n^{\prime}+1\right)+p-\ell\right\} / n^{\prime}=q .
$$

In addition, we define the dimensionless flow rate $Q_{0}(n)$ to be given by Equation (3.11) and we determine $Q_{0}$ as a function of the power-law coefficients $p_{0}, w_{0}$ and $L_{0}$ from Equation (3.12):

$$
\begin{aligned}
Q_{D}(n) & =\left\{-W_{D}^{\left(2 n^{\prime}+1\right)} \frac{d P_{D}}{d n}\right\}^{1 / n^{\prime}} . \\
Q_{0} & =\frac{H}{6}\left\{\frac{Y_{Q} W_{0}^{\left(2 n^{\prime}+1\right)} P_{0}}{2 K_{a} L_{0}}\right\}^{1 / n^{\prime}} .
\end{aligned}
$$


The fluid leak-off rate law given by Equation (2.24a) is next recast in the form of Equation (3.13) by using Equations (3.2) and (2.24b) to express $\tau$ as a function of $n$ and $t$ :

$$
\begin{aligned}
q_{L}(x, t) & =c\left(1-n^{1 / \ell}\right)^{-m} t^{-m}, \\
\tau(x) & =n^{1 / l} t .
\end{aligned}
$$

Comparing Equation (3.13) with the similarity form of Equation (3.6), we make the following identifications:

$$
\begin{aligned}
q_{L D}(n) & =\left(1-n^{1 / 2}\right)^{-m}, \\
r & =-m, \\
q_{L O} & =c .
\end{aligned}
$$

Under the similarity coordinate transformation, Equation (2.10) for conservation of fluid mass transforms to Equation (3.18):

$$
Y_{W} H W_{0}\left\{\omega W_{D}-\ln \frac{d W_{D}}{d \eta}\right\} t^{\omega-1}+\frac{Q_{0}}{L_{0}} \frac{d Q_{D}}{d \eta} t^{q-\ell}+2 Y_{L} H q_{L o} q_{L D} t^{r}=0 .
$$

Equation ( 3.18 ) can be rewritten in the form:

$$
\alpha\left\{w W_{D}(n)-\ln \frac{d W_{D}(n)}{d n}\right\} t^{\omega+l-q-1}+\frac{d Q_{D}(n)}{d n}+B q_{L D}(n) t^{I+l-q}=0 .
$$

that is suitable for evaluating the relative magnitudes of the storage and leak-off terms. The dimensionless ordinary differential equations that govern the dimensionless similarity variables under different assumptions regarding the relative importance of the storage, transport and leak-off terms can be derived from Equation (3.19). For example, when all three terms are significant, the similarity solutions are governed by Equation (3.20a) provided the power-law exponents $\ell, \omega, q$ and $r$ satisfy the compatibility relations given by Equations (3.20b) and (3.20c): 


$$
\begin{aligned}
\alpha\left\{\omega W_{D}(n)-\ell n \frac{d W_{D}(n)}{d n}\right\} & +\frac{d Q_{D}(n)}{d n}+B q_{L D}(n)=0, \\
\rho+\ell & =1+q, \\
\ell & =q-r .
\end{aligned}
$$

In Equation (3.20a) the parameters $\alpha$ and $B$ are dimensionless parameters that measure the importance of the fluid storage and fluid leak-off terms, respectively, relative to the fluid transport term. The fluid transport term is assumed to be significant when the fracture extends but it may not be the most significant term if the fracture ceases to propagate. This can occur as a result of a stress barrier, a stiffness or fracture toughness barrier, or a sand-out condition within the fracture that prevents the fracturing fluid from approaching the fracture tip. The parameters $\alpha$ and $B$ are defined by Equations (3.2la) and (3.21b), respectively:

$$
\begin{aligned}
& \alpha=Y_{G} H L_{0} W_{0} / Q_{0}, \\
& B=2 \gamma_{L} H L_{0} q_{L o} / Q_{0} .
\end{aligned}
$$

In Equation (3.20) only one of the two dimensionless parameters $\alpha$ and $B$ can be independently specified, the other is determined by the solution itself in establishing the position of the moving fracture front. We deliberately introduced this problem-determined parameter into the governing differential equation by choosing to solve the governing differential equation over the domain $0 \leq n \leq 1$ instead of over a domain of unknown extent $0 \leq \eta^{\prime} \leq \eta_{L}$. The latter domain would require a different similarity variable $\eta^{\prime}$ where $\eta^{\prime}=n_{L}$ is the position of the tip of the fracture that must be found as a part of the solution. In the general case in which storage, transport and leak-off terms are all taken into account, the dimensionless parameter $B$ is related to $\alpha$ by an expression of the form $B=2 \gamma_{L}\left(\alpha / \gamma_{W}\right)^{g\left(n^{\prime}\right)}$ where $g\left(n^{\prime}\right)$ is a known function of $n^{\prime}$ (i.e. Equations (3.55a) and $(3.59 a)$ with $(1+r-w)=0$ ). 
It will be shown that similarity solutions of the kind considered here can be found for this general case only for a certain specific value of the fluid injection power-law exponent $q$ that is related to the fluid viscosity exponent $n^{\prime}$ and leak-off exponent $m$. This limits the usefulness of the similarity solution when all three terms are important to this specific case. However, because limiting forms of the solutions, that are not subject to the above restriction on the fluid injection power-law exponent, can be found when either fluid storage or fluid leak-off are neglected, it is useful to develop the governing equations and solutions for these two limiting conditions as well. The governing Equation (3.22a) for the case when leak-off effects are small compared to storage effects is obtained by neglecting the leak-off term in Equation (3.19):

$$
\begin{gathered}
\alpha\left\{\omega W_{D}(n)-\ln \frac{d W_{D}(n)}{d n}\right\}+\frac{d Q_{D}(n)}{d n}=0 . \\
\omega+\ell=1+q . \\
\ell \neq q-r .
\end{gathered}
$$

As indicated by Equation (3.22b) and Inequality (3.22c), only the power-law balance between the fluid storage and fluid transport terms is enforced in this case, with the result that $B$ is a dimensional parameter while $\alpha$ is a dimensionless parameter that is an implicit function of the independent power-law exponents $q$ and $n^{\prime}$. The parameter $B$ must be determined as a part of the solution to Equation (3.22a). The time period over which this approximation is valid is found by requiring that:

$$
\alpha t^{\omega+l-q-1}>>t^{r+l-q}
$$

or equivalently,

$$
t<t_{S}=\left\{\alpha / B^{1 /(1+r-w)}\right\},
$$

where $t_{s}$ is identified as the upper limit of the time period when this large storage small leak-off approximation can be invoked. 
The opposite case when fluid leak-off effects dominate the effects of fluid storage can be found similarly by neglecting the fluid storage term in Equation (3.19):

$$
\begin{gathered}
\frac{d Q_{D}(n)}{d n}+B q_{L D}(n)=0, \\
\omega+\ell \neq 1+q, \\
\ell=q-I .
\end{gathered}
$$

As before, Inequality (3.24b) and Equation (3.24c) indicate that only the power-law balance between the fluid leak-off and transport terms is enforced. Consequently, $\alpha$ is a dimensional parameter while $B$ becomes $a$ dimensionless parameter that is an implicit function of the independent power-law exponents $q, n^{\prime}$ and $m$. The parameter $\alpha$ must be determined as a part of the solution to Equation (3.24a). The time period during which this approximation is valid can be found from:

$$
\alpha t^{\omega+l-q-1}<<B t^{r+l-q},
$$

or equivalently,

$$
t>t_{L}=\{\alpha / B\}^{1 /(1+r-\omega)} \text {, }
$$

where $t_{L}$ is identified as the lower limit of the time period when this large leak-off small storage approximation can be invoked.

It is important to realize that although Equations (3.23) and (3.25) look similar, they lead to different results because $\alpha$ and $B$ switch roles as the dimensionless parameter, and the value of the dimensionless parameter is evaluated using different governing equations and power-law exponent balance relations. This will become more evident in the explicit expressions that will be presented later for the dimensional parameters. In the intermediate time range $t_{S} \leq t \leq t_{L}$, storage and leak-off terms are both important and except for certain specific combinations of $q, n^{\prime}$, and $m$, no similarity solutions of the present kind can be found. 


\subsection{Initial And Boundary Conditions For Dimensionless Governing Equations}

The initial and boundary conditions that must be satisfied by the dimensionless governing Equations (3.20a), (3.22a) and (3.24a) are that the flow rate is specified at the wellbore and that it is zero at the crack-tip. In addition, the crack must close at the crack-tip for PKN fractures while it is required to close smoothly for CGDD fractures. The initial condition is that the crack width and the crack length be zero at initial time.

\section{PKN And CGDD Fractures:}

Initial Conditions:

$$
W_{D}(\infty) \quad=p_{D}(\infty)=0 .
$$

Boundary Conditions:

$$
\begin{aligned}
Q_{D}(0) & =1, \\
W_{D}(n) & =0, n \geq 1 .
\end{aligned}
$$

Boundary Condition For CGDD Fractures:

$$
\left.\frac{d W_{D}(n)}{d n}\right|_{n=1}=0
$$

The bottomhole pressure $p_{w}(t)$ that is the focus of interest in this work is given by:

$$
P_{w}(t)=S+P_{0} P_{D}(0) t^{p}
$$


3.3 Model Specific Analyses of Constant Height Fractures For PKN And CGDD Fractures

The analysis up to this point has been the same for both PKN and CGDD fractures and, in fact, is valid for constant height fractures of arbitrary cross-sectional shape. The essential difference between the PKN and CGOD models is embedded in the crack-opening relation between the crack width $W(x, t)$ and the fluid pressure $p(x, t)$. By substituting the similarity representations for these two quantities in the crack-opening relation for PKN fractures given by Equation (2.20) we arrive at:

$$
\begin{aligned}
w_{D}(n) & =p_{D}(n), \\
\omega & =p, \\
w_{0} & =H p_{0} / E_{C},
\end{aligned}
$$

so that the dimensionless similarity distributions and the power-law variations with respect to time at a fixed value of the similarity coordinate $n$ are the same for both fracture width and fluid pressure.

The corresponding relations for CGDD fractures are less straightforward. They are obtained by substituting the similarity representations for $W(x, t)$ and $p(x, t)$ in Equation (2.21):

$$
\begin{aligned}
W_{D}(n) & =\frac{2}{\pi} \int_{n}^{1} \int_{0}^{\zeta} \frac{\zeta P_{D}(\xi)}{\sqrt{\left(\zeta^{2}-\eta^{2}\right) V\left(\zeta^{2}-\xi^{2}\right)} d \xi d \zeta,} \\
\omega & =p+\ell, \\
W_{0} & =2 L_{0} p_{0} / E_{c} .
\end{aligned}
$$

We see from Equation (3.35), that unlike in the case of PKN fractures, the growth exponents for fracture width and fluid pressure of CGDD fractures are different by an amount equal to the growth exponent for the length of the fracture. This basic feature is the reason for the radically different pressure responses exhibited by PKN and CGDD fractures. 
The condition of smooth closure of the fracture at its tip can be either directly imposed on $W_{D}(n)$ as in Equation (3.29) or indirectly imposed on $P_{D}(\eta)$ as in Equation (3.37), using the result of Equation (2.22a):

$$
\int_{0}^{1} \frac{P_{D}(n)}{r\left(1-n^{2}\right)} d n=0
$$

\subsubsection{Power-Law Exponents}

The power-law exponents for fracture width, fluid pressure and fracture length can now be obtained in terms of $q, n^{\prime}$ and $m$ by solving Equations (3.10), (3.16), (3.20b) and (3.20c) together with either Equation (3.32) for PKN fractures or Equation (3.35) for CGDD fractures. Equation (3.20c) must be not be enforced for the limiting case of small leak-off effects while Equation (3.20b) must not be used in the limiting case of small storage effects. When these Equations are solved simultaneously we obtain the following results for the power-law exponents:

\section{General Case}

\section{PKN FRACTURES}

$p=\frac{1}{2 n^{\prime}+3}+\frac{n^{\prime}+1}{2 n^{\prime}+3} q=1 / 5$ for $n^{\prime}=1, q=0$,

$\omega=\frac{1}{2 n^{\prime}+3}+\frac{n^{\prime}+1}{2 n^{\prime}+3} q=1 / 5$ for $n^{\prime}=1, q=0$,

$\ell=\frac{2 n^{\prime}+2}{2 n^{\prime}+3}+\frac{n^{\prime}+2}{2 n^{\prime}+3} q=4 / 5$ for $n^{\prime}=1, q=0$,

$q=2-\frac{2 n^{\prime}+3}{n^{\prime}+1} m=3 / 4$ for $n^{\prime}=1, m=1 / 2$. 


\section{CGDD FRACTURES}

$p=\frac{n^{\prime}}{n^{\prime}+2} \quad=-1 / 3$ for $n^{\prime}=1, q=0$,

$\omega=\frac{n^{\prime}}{n^{\prime}+2}+\frac{1}{2} q=1 / 3$ for $n^{\prime}=1, q=0$,

$\ell=\frac{n^{\prime}+1}{n^{\prime}+2}+\frac{1}{2} q=2 / 3$ for $n^{\prime}=1, q=0$,

$q=\frac{2 n^{\prime}+1}{n^{\prime}+2}-2 m=0$ for $n^{\prime}=1, m=1 / 2$.

These results indicate that for fluid injection at constant flow rate (i.e. $q=0$ ) fracture width at any fixed value of $n$, and in particular at $n=0$ at the wellbore, increases (i.e. $\omega>0$ ) for both PKN and CGDD fractures as the fracture extends (i.e. $l>0$ ) with time. The rate of increase of the fracture width is generally smaller for PKN fractures than for CGDD fractures. However, the pressure at a fixed value of $n$ increases (i.e.p $>0$ ) with time in the case of PKN fractures but decreases (i.e. $p<0$ ) with time in the case of CGDD fractures yielding a significant qualitative difference in the bottomhole pressure response. Further, for both types of fractures these results predict smaller rates of increase or decrease for Newtonian fluids $\left(n^{\prime}=1\right)$ than for non-Newtonian power-law fluids that have smaller values of $n^{\prime}$.

The apparently strange results of Equations (3.41) and (3.45) in which the injection flow rate exponent $q$ is given as functions of $n$ ' and merely reflect the fact that power-law similarity solutions of this kind can be obtained for the general case when all three storage, transport and leak-off terms are retained only for the specific value of the pumping rate exponent $q$ that satisfies this Equation for given $n^{\prime}$ and $m$. For example, for the case of a Newtonian fluid $\left(n^{\prime}=1\right)$ and the standard half-power $(m=1 / 2)$ leak-off law, Equation (3.35) for PKN fractures is satisfied only if $q=3 / 4$ and Equation (3.39) for CGDD fractures is satisfied only if $q=0$. Therefore, for general analysis of hydraulic fracturing considering storage, transport and leak-off terms simultaneously, these similarity 
solutions are not very useful unless the values of $n$ ' and $m$ are chosen to satisfy these equations. However, as we will show, they remain useful for interpreting the limiting cases of small leak-off when the rate of fluid leak-off is negligible compared to rate of change in fluid storage, and for interpreting the opposite case of large rate of fluid leak-off when fluid leak-off dominates the rate of change in fluid storage. For these limiting cases there is no restriction on the pumping rate power-law exponent $q$.

\section{Case of Small Fluid Leak-off}

The results for this case are obtained by not enforcing Equation (3.20c) that represents the compatibility of power-law behaviors between the fluid leak-off term and the fluid transport term in Equation (3.18). When Equation (3.20c) is neglected, the results that are obtained for $p$, $\omega$ and $\ell$ are identical to those given above by Equations (3.38), (3.39) and (3.40) for PKN fractures and by Equations (3.42), (3.43) and (3.44) for CGDD fractures. No Equations that correspond to Equations (3.41) and (3.45) are then obtained.

\section{Case of Small Fluid Storage}

Similarly, the results for the case when fluid leak-off dominates fluid storage effects are obtained by not enforcing Equation (3.20b) that represents the compatibility of the power-law behaviors between the fluid storage term and the fluid transport term in Equation (3.18). These results are given below:

\section{PKN FRACTURES}

$$
\begin{aligned}
& p=\frac{m}{2 n^{\prime}+2}+\frac{1}{2} q=1 / 8 \text { for } n^{\prime}=1, q=0, m=1 / 2, \\
& \omega=\frac{m}{2 n^{\prime}+2}+\frac{1}{2} q=1 / 8 \text { for } n^{\prime}=1, q=0, m=1 / 2, \\
& \ell=m+q=1 / 2 \text { for } n^{\prime}=1, q=0, m=1 / 2,
\end{aligned}
$$




\section{CGDD FRACTURES}

$p=-\frac{n^{\prime} m}{n^{\prime}+1}-\frac{n^{\prime}}{2 n^{\prime}+2} q=-1 / 4$ for $n^{\prime}=1, q=0, m=1 / 2$,

$\omega=\frac{m}{n^{\prime}+1}+\frac{n^{\prime}+2}{2 n^{\prime}+2} q=1 / 4$ for $n^{\prime}=1, q=0, m=1 / 2$,

$\ell=m+q=1 / 2$ for $n^{\prime}=1, q=0, m=1 / 2$,

\subsubsection{Power-Law Coefficients}

The power-law coefficients $p_{0}, W_{0}$ and $L_{0}$ can be determined as functions of $Q_{0}, E_{C}, C, H, K^{\prime}, n^{\prime}$ and $\alpha$ and/or $B$. When the general case is being solved, $\alpha\left(n^{\prime}, m, B\right)$ will be considered to be determined by the solution as a function of $n^{\prime}, m$ and $B$. In the limiting case of small fluid leak-off, the power-law coefficients depend only on the dimensionless parameter $\alpha\left(q, n^{\prime}\right)$; while in the limiting case of small fluid storage they depend only on the dimensionless coefficient $B\left(q, n^{\prime}, m\right)$. The expressions that are given below for these coefficients were obtained by simultaneously solving Equations (3.12), (3.21a), (3.21b) and either Equation (3.33) for PKN fractures or Equation (3.36) for CGDD fractures. Equations (3.55a) and (3.59a) relate the dimensional parameter $B$ to the dimensionless parameter $\alpha$ for PKN and CGDD fractures in terms of the characteristic times ${ }^{T_{P}}$ and ${ }^{{ }} \mathrm{C}$ defined by Equations (3.55b) and (3.59b), respectively. Note that in Equations (3.55a) and (3.55b), $(1+r-w)=0$ for this general case. The estimates for $t_{S}$ and $t_{L}$, given below, show that the times $\tau_{P}$ and ${ }^{{ }} \mathrm{C}$ characterize the nature of the solution and its transition from primarily fluid storage dominated fracture extension at small times to primarily leak-off dominated fracture extension at large times. Note that these characteristic times are different for PKN and CGDD models but are the same for both limiting conditions. 
General Case

\section{PKN FRACTURES}

$p_{0}=\left[\frac{2.6^{n^{\prime}}}{\gamma_{Q}} \frac{\alpha}{\gamma_{W}} K_{a} E_{c}^{\left(2 n^{\prime}+2\right)} H^{-\left(3 n^{\prime}+3\right)} Q_{0}\left(n^{\prime}+1\right)\right]^{1 /\left(2 n^{\prime}+3\right)}$,

$W_{0}=\left[\frac{2.6^{n^{\prime}}}{Y_{Q}} \frac{\alpha}{\gamma_{W}} K_{a} E_{c}^{-1} H^{-n^{\prime}} Q_{0}\left(n^{\prime}+1\right)\right]^{1 /\left(2 n^{\prime}+3\right)}$,

$L_{0}=\left[\frac{2.6^{n^{\prime}}}{\gamma_{Q}} \frac{\alpha}{\gamma_{W}}\left(2 n^{\prime}+2\right) K_{a}^{-1} E_{c} H^{-\left(n^{\prime}+3\right)} Q_{0}\left(n^{\prime}+2\right)\right]^{1 /\left(2 n^{\prime}+3\right)}$,

$\left(B / 2 \gamma_{L}\right)=\left(\alpha / \gamma_{W}\right)^{\left(2 n^{\prime}+2\right) /\left(2 n^{\prime}+3\right)} \tau_{P}-(1+\Gamma-\omega)$,

$\tau_{P}=\left[\frac{2.6^{n^{\prime}}}{Y_{Q}} \frac{K_{a} Q_{0}^{\left(n^{\prime}+1\right)}}{c^{-\left(2 n^{\prime}+3\right) H^{n^{\prime} E_{E}}}}\right]^{\left\{2 n^{\prime}+2-\left(2 n^{\prime}+3\right) m-\left(n^{\prime}+1\right) q\right\}}$.

\section{CGDD FRACTURES}

$p_{0}=\left[\frac{4.6^{n^{\prime}}}{Y_{Q}} \frac{\alpha^{-n^{\prime}}}{Y_{W}^{-n^{\prime}}} K_{a} E_{c}^{\left(n^{\prime}+1\right)}\right]^{1 /\left(n^{\prime}+2\right)}$,

$W_{0}=\left[\frac{4.6^{n^{\prime}}}{Y_{Q}} \frac{\alpha^{2}}{Y_{W}^{2}} K_{a_{c} E^{-1} H^{-\left(n^{\prime}+2\right)} Q_{Q_{0}}\left(n^{\prime}+2\right)}\right]^{1 / 2\left(n^{\prime}+2\right)}$,

$L_{0}=\left[\frac{\left(4.6^{n^{\prime}}\right)^{-1}}{Y_{Q}^{-1}} \frac{\alpha^{\left(2 n^{\prime}+2\right)}}{Y_{W}^{\left(2 n^{\prime}+2\right)}} K_{a}^{-1} E_{c^{-}} H^{-\left(n^{\prime}+2\right)} Q_{0}{ }^{\left(n^{\prime}+2\right)}\right]^{1 / 2\left(n^{\prime}+2\right)}$,

$\left(B / 2 \gamma_{L}\right)=\left(\alpha / \gamma_{W}\right)^{\left(n^{\prime}+1\right) /\left(n^{\prime}+2\right)} \tau_{C}^{-(1+r-\omega)}$,

${ }^{T} C=\left[\frac{4.6^{n^{\prime}}}{Y_{Q}} \frac{K_{a} Q_{0}^{\left(n^{\prime}+2\right)}}{c^{\left(2 n^{\prime}+4\right){ }_{H}\left(n^{\prime}+2\right)} E_{c}}\right]^{\left\{2 n^{\prime}+2-\left(2 n^{\prime}+4\right) m-\left(n^{\prime}+2\right) q\right\}}$. 


\section{Case of Small Fluid Leak-off}

The power-law coefficients when leak-off is negligible are identical to those given by Equations (3.52), (3.53) and (3.54) for PKN fractures and by Equations (3.56), (3.57) and (3.58) for CGDD fractures. The dimensional coefficient $\alpha$ can be calculated for PKN and CGDD fractures from Equations (3.55) and (3.59), respectively. The time limit $t_{s}$ for the small leak-off assumption can be computed using a from the solution to the governing equation and the value of $B$ given by Equation (3.55a) (for PKN fractures) and Equation (3.59a) (for CGDD fractures) in the following Equations:

$$
\begin{aligned}
& \text { PKN FRACTURES } \\
& t_{S}=\tau_{P}\left[\left(\alpha / \gamma_{W}\right)^{1 /\left(2 n^{\prime}+3\right)}\left(\gamma_{W^{\prime}} / 2 \gamma_{L}\right)\right]^{1 /(1+r-w)} \\
& 1+r-\omega=\left[\frac{2 n^{\prime}+2}{2 n^{\prime}+3}-\frac{n^{\prime}+1}{2 n^{\prime}+3} q-m\right] . \\
& \text { CGDD FRACTURES } \\
& t_{S}=\tau_{P}\left[\left(\alpha / \gamma_{W}\right)^{1 / 2\left(2 n^{\prime}+2\right)}\left(\gamma_{W} / 2 \gamma_{L}\right)\right]^{1 /(1+r-\omega)} \\
& 1+r-\omega=\left[\frac{n^{\prime}+1}{n^{\prime}+2}-\frac{1}{2} q-m\right] .
\end{aligned}
$$

\section{Case of Small Fluid Storage}

The power-law coefficients when fluid storage is negligible are given by Equations (3.62), (3.63) and (3.64) for PKN fractures and by Equations (3.66), (3.67) and (3.68) for CGDD fractures. The dimensional coefficient $\alpha$ can be calculated for PKN and CGDD fractures from Equations (3.65) and (3.69), respectively. 


\section{PKN FRACTURES}

$p_{0}=\left[\frac{2.6^{n^{\prime}}}{q_{Q}} \frac{B}{2 \gamma_{L}} K_{a} C^{-1} E_{c}\left(2 n^{\prime}+1\right) H_{H^{-}}\left(3 n^{\prime}+2\right) Q_{0}\left(n^{\prime}+1\right)\right]^{1 /\left(2 n^{\prime}+2\right)}$,

$W_{0}=\left[\frac{2.6^{n^{\prime}}}{\gamma_{Q}} \frac{B}{2 \gamma_{L}} K_{a} C^{-1} E_{c}{ }^{-1} H^{-n^{\prime}} Q_{0}{ }^{\left(n^{\prime}+1\right)}\right]^{1 /\left(2 n^{\prime}+2\right)}$,

$L_{0}=\left[\frac{B}{2 r_{L}} C^{-1} H^{-1} Q_{0}\right]$,

$\left(\alpha / \gamma_{W}\right)=\left(B / 2 \gamma_{L}\right)^{\left(2 n^{\prime}+3\right) /\left(2 n^{\prime}+2\right)} \tau_{P}{ }^{(1+r-\omega)}$,

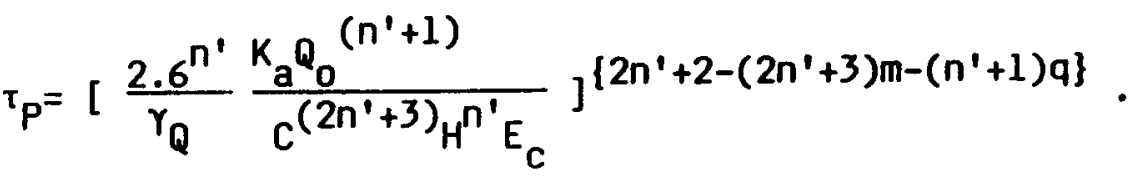

\section{CGDD FRACTURES}

$P_{0}=\frac{1}{2}\left[\frac{4.6^{n^{\prime}}}{\gamma_{Q}} \frac{B^{-2 n^{\prime}}}{\left(2 \gamma_{L}\right)^{-2 n^{\prime}}} K_{a} c^{2 n^{\prime} E_{c}}{ }^{\left(2 n^{\prime}+1\right)} H^{n^{\prime}} Q_{0}{ }^{-n^{\prime}}\right]^{1 / 2\left(n^{\prime}+1\right)}$,

$W_{0}=\left[\frac{4.6^{n^{\prime}}}{\gamma_{Q}} \frac{B^{2}}{\left(2 \gamma_{L}\right)^{2}} K_{a} C^{-2} E_{c}{ }^{-1} H^{-\left(n^{\prime}+2\right)} Q_{0}-\left(n^{\prime}+2\right)\right]^{1 / 2\left(n^{\prime}+1\right)}$,

$L_{0}=\left[\frac{B}{2 r_{L}} c^{-1} H^{-1} Q_{0}\right]$,

$\left(\alpha / \gamma_{W}\right)=\left(B / 2 \gamma_{L}\right)^{\left(n^{\prime}+2\right) /\left(n^{\prime}+1\right)} T_{C}{ }^{(1+\Gamma-\omega)}$,

${ }^{T} C=\left[\frac{4.6^{n^{\prime}}}{r_{Q}} \frac{k_{a_{0}}{ }^{\left(n^{\prime}+2\right)}}{C^{\left(2 n^{\prime}+4\right)} H^{\left(n^{\prime}+2\right)} E_{C}}\right]^{\left\{2 n^{\prime}+2-\left(2 n^{\prime}+4\right) m-\left(n^{\prime}+2\right) q\right\}}$.

The time limit $t_{L}$ for the small storage assumption can be computed by substituting in the following equations the value of $B$ from the 
solution to the governing equation and the value of $\alpha$ given by Equation (3.65a) (for PKN fractures) or Equation (3.69a) (for CGDD fractures):

\section{PKN FRACTURES}

$$
\begin{aligned}
t_{L} & =\tau_{P}\left[\left(B / 2 \gamma_{L}\right)^{1 /\left(2 n^{\prime}+2\right)}\left(\gamma_{W} / 2 \gamma_{L}\right)\right]^{1 /(1+r-\omega)} \\
1+\Gamma-\omega & =\left[1-\frac{1}{2} q-\frac{2 n^{\prime}+3}{2 n^{\prime}+2} m\right] .
\end{aligned}
$$

\section{CGDD FRACTURES}

$$
\begin{aligned}
t_{L} & =\tau_{P}\left[\left(B / 2 \gamma_{L}\right)^{1 / 2\left(n^{\prime}+1\right)}\left(q_{W} / 2 q_{L}\right)\right]^{1 /(1+r-w)}, \\
1+r-w & =\left[1-\frac{n^{\prime}+2}{2 n^{\prime}+2} q-\frac{n^{\prime}+2}{2 n^{\prime}+2} m\right] .
\end{aligned}
$$

\subsubsection{Values of The Dimensionless Parameters $\alpha$ and $B$}

\section{Case of Small Fluid Leak-off}

The dimensionless parameter $\alpha$ for negligible fluid leak-off and the dimensional parameter $B$ for negligible fluid storage must be computed as a part of the solution to the boundary-initial value problems that were previously formulated for these conditions. These boundary-initial value problems are difficult to solve, particularly for CGOD fractures which involve the solution of an integro-differential equation, and are beyond the scope of this work. However, for PKN fractures, calculations equivalent to those required here were performed by Nordgren [28] for the case of a Newtonian fluid $\left(n^{\prime}=1\right)$ and fluid injection at a constant flowrate $(q=0)$. Nordgren posed his boundary value problem in terms of a similarity variable that varied from zero at the wellbore to an unknown value at the fracture tip. This unknown value was determined as a part of the solution to the problem. Because this approach is fully equivalent to the boundary fixing method employed in the present work, the corresponding value of a can be determined from Nordgren's solution by comparing the value for the fracture length power-law coefficient $L_{0}$ from his Equation (C-9) with that given by Equation (3.54): 


$$
\begin{gathered}
{\left[\frac{2.6^{n^{\prime}}}{Y_{Q}} \frac{\alpha^{\left(2 n^{\prime}+2\right)}}{Y_{W}\left(2 n^{\prime}+2\right)}\right]^{1 /\left(2 n^{\prime}+3\right)}=0.68,} \\
\alpha=0.228,
\end{gathered}
$$

for $n^{\prime}=1, Y_{Q}=3 \pi / 16$ and $Y_{W}=\pi / 4$.

For CGDD fractures no reliable values of $\alpha$ can be obtained from previous work in the literature. Geertsma and De Klerk [9] present an approximate power-law expression for $L(t)$ but the assumptions invoked to derive these results are too restrictive to be either meaningful or reliable for evaluating $\alpha$. For example, Equation (23) in their paper has been derived assuming that the fluid pressure profile can be evaluated by neglecting the storage term in the equation of mass conservation and by using the value for fracture width at the wellbore that is obtained for a fracture subjected to a uniform pressure equal to (what amounts to) the average fluid pressure in the fracture. If, regardless of our reservations about the accuracy of this approach, this Equation is used to compute a by comparing against the power-law coefficient $L_{0}$ given by Equation (3.58), we obtain:

$$
\begin{gathered}
{\left[\frac{Y_{Q}}{4.6^{n^{\prime}}} \frac{\alpha^{\left(2 n^{\prime}+2\right)}}{Y_{W}^{\left(2 n^{\prime}+2\right)}}\right]^{1 / 2\left(n^{\prime}+2\right)}=0.68,} \\
\alpha=1.51,
\end{gathered}
$$

for $n^{\prime}=1$ and $\gamma_{Q}=\gamma_{W}=1$. Note that if an elliptical cross-section is assumed for this CGDD fracture, then $Y_{Q}=3 \pi / 16, \gamma_{W}=\pi / 4$ yielding $\alpha=1.35$.

\section{Case of Small Fluid Storage}

In the case of small fluid storage, the value of the dimensionless parameter $B$ is easily obtained by integrating Equation (3.24a) subject to the boundary conditions given by Equations (3.27) and (3.28): 


$$
\begin{aligned}
Q_{D}(\eta) & =B \int_{\eta}^{1} q_{L D}(\xi) d \xi, \\
B & =1 /\left[\int_{0}^{1} q_{L D}(\xi) d \xi\right] .
\end{aligned}
$$

For $n^{\prime}=1$ and $m=1 / 2$, Equations (3.76) and (3.77) yield the standard results:

$$
\begin{aligned}
Q_{D}(n) & =1-\frac{2}{\pi} \sin ^{-1} \eta, \\
B & =2 / \pi .
\end{aligned}
$$

The above results are valid for both PKN and CGDD fractures. The dimensionless width and dimensionless pressure profiles for PKN fractures can be obtained by substituting these expressions in the definition of $Q_{D}(n)$ given by Equation (3.11) and integrating once more:

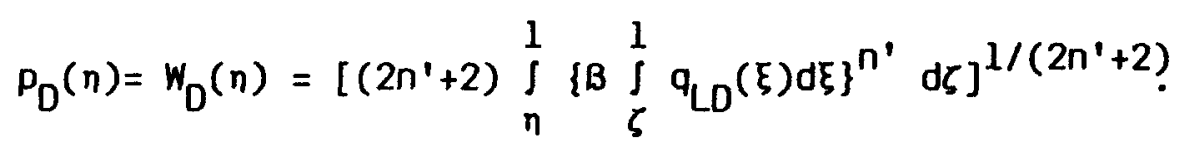

For $n^{\prime}=1$ and $m=1 / 2$, Equation (3.80) gives:

$$
\begin{gathered}
P_{D}(n)=W_{D}(n)=\left[\frac{8}{\pi} v\left(1-\eta^{2}\right)-4 n\left(1-\frac{2}{\pi} \sin ^{-1} \eta\right)\right]^{1 / 4}, \\
P_{D}(0)=W_{D}(0)=(8 / \pi)^{1 / 4}=1.263 .
\end{gathered}
$$

To obtain the width and pressure profiles corresponding to Equation (3.81) for CGDD fractures it is necessary to solve an integro-differential equation for either the dimensionless width $W_{D}(n)$ or the dimensionless pressure $p_{D}(n)$. This is a difficult task that is not attempted here. 


\section{EXTENDED ANALYSIS FOR FRACTURES WITH ARBITRARY ASPECT RATIO}

\subsection{The Hybrid CGDO-PKN Fracture Model}

Let us now consider the possibility of generalizing the foregoing results for CGDD fractures of uniform width to the case of fractures with non-uniform width cross-sections, and in particular to cross-sections of elliptical shape as in PKN fractures. The basic difference between PKN and CGDD fractures lies in the neglect of the contribution to the crack-opening stiffness from either the lateral or the longitudinal direction based on estimates of their relative magnitudes. For fractures that do not slip at the fracture boundary, the crack-opening stiffness from a given direction is a function of the curvature of the fracture width profile in that direction so that the contributions to stiffness from these two directions depend on the relative magnitudes of the height $H$ and the length $L(t)$. Therefore, we can extend the results for CGDD fractures to arbitrary cross-sections that do not slip at the ends provided the ratio $L(t) / H$ is sufficiently small. Likewise, PKN fracture assumptions are valid only for fractures for which the ratio $L(t) / H$ is sufficiently large. The assumption of a uniform width cross-section with slip at the top and bottom boundaries is now seen to be really necessary for CGOD fractures only if we insist on applying this model for arbitrarily large $L(t) / H$ ratios much greater than unity. Similarly, it is seen that the PKN fracture model cannot be valid for arbitrarily small $L(t) / H$ much smaller than unity because the stiffness in the longitudinal direction would begin to dominate for sufficiently small $L(t)$ at a fixed value of $H$.

\subsubsection{Characteristic Times For Fracture Extension; PKN And CGOD Fractures}

These observations imply that, subject to the assumptions that the fracture does not slip at its edges and that the fracture height remains constant and uniform at all times, the CGDD fracture model would be applicable to the propagation of hydraulic fractures at early times such 
that $L(t) / H \ll 1$ and the PKN fracture model would be applicable at late times such that $L(t) / H \gg 1$. To determine at what point in time we should switch from the CGDD model to the PKN model when attempting to predict the extension of a fracture from large to small values of $L(t) / H$ let us restrict the aspect ratio of CGDD fractures with non-slipping lateral fracture edges to:

$$
1 \gg L(t) / H \geq 0 ; t_{H L C} \gg t \geq 0 \text {, }
$$

and the aspect ratio of PKN fractures to:

$$
\infty>L(t) / H \gg 1 ; \infty>t \gg t_{H L P}
$$

and determine the times $t_{H L C}$ and $t_{H L P}$ that define the time ranges within which these aspect ratio restrictions are satisfied by each model. If these times overlap, then any time within the overlap region should be satisfactory for switching from the CGDD model to the PKN model as the aspect ratio increases beyond unity with fracture extension. On the other hand, if $t_{H L C}$ is much less than $t_{H L P}$ and a substantial gap exists between them, then a transition model that simultaneously allows for stiffness contributions from both directions would be required to bridge the time regime when the aspect ratio is in the neighborhood of unity. Intuitively, it would seem that such a transition model would always be needed because of the coarseness of our transition time estimates. These estimates can be refined by detailed calculations using the full governing equations.

For this analysis, it is convenient to recast the power-law growth expressions for fracture length, for small fluid leak-off and small fluid storage, in dimensionless form in terms of the aspect ratio $L_{D}=L(t) / H$ and the dimensionless times $t_{D S}=t / \tau_{H L S}$ and $t_{D L}=t / \tau_{H L L}$ Note that the characteristic time ${ }^{T H L S}$ for the small leak-off model and $\tau_{H L L}$ for the small storage model are different for these two regimes; but, unlike ' $P$ and ' $C$ ' they are the same for both PKN and CGDD models for the same time regime. 
Case of Small Fluid Leak-off

$$
\begin{aligned}
& \tau_{H L S}=\left[\frac{K_{a} H^{\left(3 n^{\prime}+6\right)}}{E_{c} Q_{0}^{\left(n^{\prime}+2\right)}}\right]^{1 /\left[2 n^{\prime}+2+\left(n^{\prime}+2\right) q\right]} . \\
& \text { PKN FRACTURES } \\
& L_{D}=\left[\frac{2.6^{n^{\prime}}}{r_{Q}} \frac{\alpha^{\left(2 n^{\prime}+2\right)}}{r_{W}^{\left(2 n^{\prime}+2\right)}}\right] t_{D S}{ }^{l_{P}} \text {, } \\
& \ell_{P}=\frac{2 n^{\prime}+2}{2 n^{\prime}+3}+\frac{n^{\prime}+2}{2 n^{\prime}+3} q . \\
& \text { CGOD FRACTURES } \\
& L_{D}=\left[\frac{\gamma_{Q}}{4.6^{n^{\prime}}} \frac{\alpha^{\left(2 n^{\prime}+2\right)}}{\gamma_{W}{ }^{\left(2 n^{\prime}+2\right)}}\right] t_{D S}{ }^{l_{C}} \text {, } \\
& \ell_{C}=\frac{n^{\prime}+1}{n^{\prime}+2}+\frac{1}{2} q .
\end{aligned}
$$

\section{Case of Small Fluid Storage}

$$
\tau_{H L L}=\left[\frac{H^{2} C}{Q_{0}}\right]^{1 /(m+q)} .
$$

PKN FRACTURES

$$
L_{D}=\left[\frac{B}{2 \gamma_{L}}\right] t_{D L}^{(m+q)} \text {. }
$$

\section{CGDD FRACTURES}

$$
L_{D}=\left[\frac{B}{2 q_{L}}\right] t_{D L}^{(m+q)} \text {. }
$$

\subsubsection{Aspect Ratio Limiting Times}

The parameters $\tau_{H L S}$ and $\tau_{H L L}$ defined by Equations (4.3) and (4.6) for the small fluid leak-off and small starage limiting conditions 
are the characteristic times for fracture propagation over a distance $H$, and are, therefore, useful indicators of the rapidity of fracture extension. By setting $L_{D}=1$ in Equations (4.4a), (4.5a), (4.7) and (4.8), we obtain the following estimates for the aspect ratio limiting times $t_{H L P}$ and $t_{H L C}$ :

\section{Case of Small Fluid Leak-off}

$$
\begin{aligned}
& t_{H L P}=\left[\frac{2.6^{n^{\prime}}}{\gamma_{Q}} \frac{\alpha^{\left(2 n^{\prime}+2\right)}}{\gamma_{W}^{\left(2 n^{\prime}+2\right)}}\right]^{-1 / \ell_{P}} \tau_{H L S^{\prime}} \\
& t_{H L C}=\left[\frac{\gamma_{Q}}{4.6^{n^{\prime}}} \frac{\alpha^{\left(2 n^{\prime}+2\right)}}{\gamma_{W}^{\left(2 n^{\prime}+2\right)}}\right]^{-1 / l_{C}} \tau_{H L S^{\prime}}
\end{aligned}
$$

\section{Case of Small Fluid Storage}

$$
\begin{aligned}
& t_{H L P}=\left[\frac{B}{2 \gamma_{L}}\right]^{-1 /(m+q)} \tau_{H L L}, \\
& t_{H L C}=\left[\frac{B}{2 \gamma_{L}}\right]^{-1 /(m+q)} \tau_{H L L},
\end{aligned}
$$

It can shown that the overall characteristic time $\tau_{p}$ for PKN-type fracture extension, and the overall characteristic time ${ }^{\top} C$ for CGDD-type fracture extension are related to the corresponding small leak-off and small storage characteristic times $\tau_{H L S}$ and $\tau_{H L L}$ for each model by:

$\tau_{P}=\left[\frac{2.6^{n^{\prime}}}{Y_{Q}} \frac{\tau_{H L S}\left\{2 n^{\prime}+2+\left(n^{\prime}+2\right) q\right\}}{\tau_{H L L}(m+q)\left(2 n^{\prime}+3\right)}\right]^{1 /\left\{2 n^{\prime}+2-\left(n^{\prime}+1\right) q-\left(2 n^{\prime}+3\right) m\right\}}$,

and

$\tau_{C}=\left[\frac{4.6^{n^{\prime}}}{Y_{Q}} \frac{\tau_{H L S}\left\{2 n^{\prime}+2+\left(n^{\prime}+2\right) q\right\}}{\tau_{H L L}(m+q)\left(2 n^{\prime}+4\right)}\right]^{1 /\left\{2 n^{\prime}+2-\left(n^{\prime}+2\right) q-\left(2 n^{\prime}+4\right) m\right\}}$, 
respectively. The above relationships between the characteristic times that were separately defined for different time regimes in apparently different models reflect the fact that PKN and CGDD models are not really different models but that they represent the limiting forms of a hybrid fracture model for arbitrary aspect ratios, as the aspect ratio varies continuously from small to large values past the aspect ratio of unity.

\subsection{Bottomhole Pressure Response of The Hybrid CGDD-PKN Fracture Model}

An idealized representation of the bottomhole pressure record that would be obtained for a constant height hydraulic fracture described by this extended hybrid CGDD-PKN model is given by curve ABCDEFG in Figure 2. The corresponding curve for fracture length is given by the curve IJKLM. The pumping of the fracturing fluid at a constant rate is begun at $A$ and this leads to a rapid rise in pressure to $B$ followed by breakdown of the formation and extension of the fracture beyond the borehole stress cage to the point $C$. Between $C$ and $D$ the fracture extends as a CGDD type fracture with the bottomhole pressure declining with time. The aspect ratio approaches one in the neighborhood of $D$ and beyond $D$ the mode of fracture extension gradually becomes of the PKN-type. As a result, the bottomhole pressure begins to increase with time as indicated by the portion $D E$ of the pressure curve. At $E$ the pumps are stopped, the flow effectively ceases in the fracture, and the fluid pressure achieves the instantaneous shut-in pressure value (ISIP) which is taken to be equal to the minimum principal stress $S$ acting to close the fracture. Due to fluid leak-off, the pressure will decline thereafter; this is indicated by the segment FG. Because of the stored energy of the fracture, fracture fluid flow and fracture extension does not actually cease immediately with the shutdown of the pumps. This is indicated by the curve for fracture length which continues to increase until point $L$ beyond the pump shutdown time. Thereafter, it does not extend and gradually begins to close as the pressure in the fracture declines with fluid leak-off. 


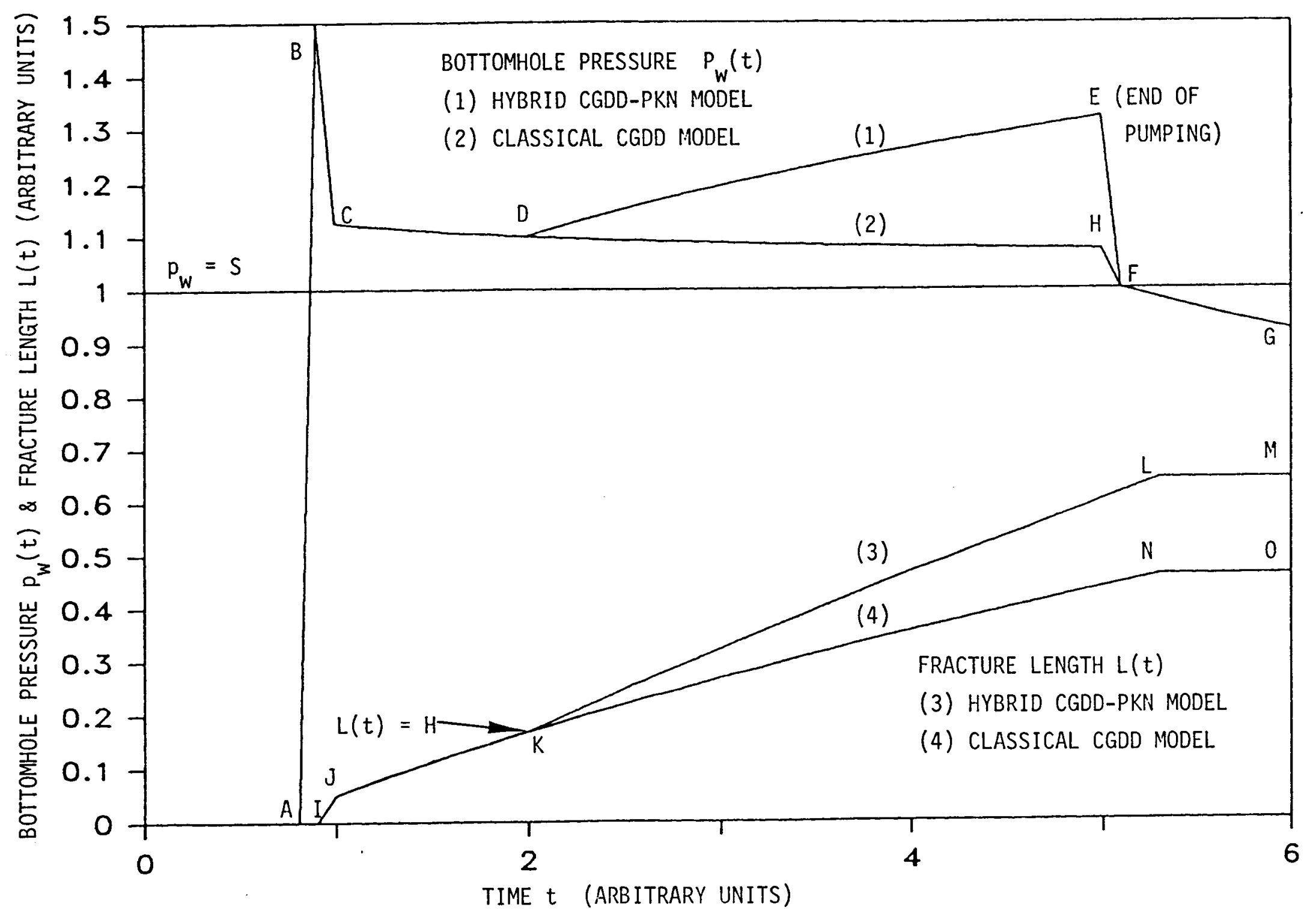

FIGURE 2 : BOTTOMHOLE PRESSURE AND FRACTURE LENGTH VARIATION WITH TIME IN IDEALIZED HYDRAULIC FRACTURING JOB. 
The bottomhole pressure curve ABCDHFG indicates how the bottomhole pressure would continue to decline past the point $D$ if the fracture continued to respond as a true CGDD fracture having a cross-section of uniform width and undergoing slip at the top and bottom edges of the fracture. The corresponding variation of fracture length with time is given by the curve IJKNO.

The increase of bottomhole pressure with time displayed by the segment DE that is associated with stable PKN-type fracture extension is often present in hydraulic fracturing pressure records. However, additional complications not indicated in Figure 2 can intervene between the point $E$ and the shutdown of the pumps. These are usually related to sand-out in the fracture, screen-out at the wellbore, stable fracture height extension or rapid unstable growth of fracture height $[26,27]$. Sand-out inhibits fracture fluid flow and fracture propagation, and leads to a sharp increase in the slope of the bottomhole pressure curve. Stable fracture extension contributes added compliance to the fracture and leads to a smaller, even zero, slope in the bottomhole pressure vs. time curve. On the other hand, rapid unstable growth of fracture height is characterized by a negative slope and decrease in the bottomhole pressure from a previously attained high. These complex phenomena are beyond the scope of the present analysis and are not dealt with here.

It is, however, of interest that a CGDD-type fracture extension event of the kind predicted by the hybrid model at early times is not often observed in hydraulic fracture pressure records even in situations where there is reason to believe that there is no substantial growth in fracture height at early times. When behavior of this kind is observed, it is usually attributed to height growth at these early times. One possible explanation is that the time range within which pressure decline due to CGDD-type fracture extension occurs is very small so that the point $D$ is too close to $\mathrm{C}$ to be separately identified, particularly because there usually is a substantial amount of short term fluctuations and noise in the pressure record immediately after the sudden breakdown of the formation. To explore this point further, let us estimate the times $t_{H L P}$ 
and $t_{H L C}$ for a hydraulic fracturing job having the parameters given in Table I. A Newtonian fluid is assumed to permit the use of the previously calculated values of $\alpha$ and $B$.

TABLE I.

Pumping Rate $Q_{0} \ldots \ldots \ldots \ldots \ldots \ldots \ldots . \ldots 10 \mathrm{bbl} / \mathrm{min}$.

Fracture Height $H \ldots \ldots \ldots \ldots \ldots \ldots . . .100 \mathrm{ft}$.

Fluid Leak-0ff Coefficient $C \ldots \ldots \ldots 0.0015 \mathrm{ft} . \mathrm{min}^{-1 / 2}$.

Flow Law Index $n^{\prime} \ldots \ldots \ldots \ldots \ldots \ldots$

Apparent Fluid Viscosity $k_{\mathrm{a}} \ldots \ldots \ldots \ldots 100 \mathrm{cp}$.

Poisson's Ratio v................ 0.15

Elastic Shear Modulus G .......... $2.6 \times 10^{6}$ psi.

These values yield the following estimates for $t_{H L P}$ and $t_{H L C}$ for $a$ fracture extending as a PKN fracture. We are not able to compute the corresponding values for a CGOD fracture because a reliable value for the dimensionless parameter $\alpha$ is not available. To obtain this value the similarity boundary value problem previously identified for this case would have to be solved.

$$
\begin{aligned}
& \tau_{H L S}=49 \mathrm{sec} . \\
& \tau_{H L L}=4.3 \mathrm{sec} . \\
& \tau_{P}=5.36 \times 10^{9} \mathrm{sec} .
\end{aligned}
$$

Estimates From Small Fluid Leak-Off Model

$$
\begin{aligned}
& t_{H L P}=11.2 \tau_{H L S}=549 \mathrm{sec} . \\
& t_{H L C}=5.15 \tau_{H L S}=252 \mathrm{sec} . \\
& t_{S}=1.04 \times 10^{8} \mathrm{sec} .
\end{aligned}
$$




\section{Estimates From Small Fluid Storage Model}

$$
\begin{aligned}
& t_{H L P}=9.86 \tau_{H L L}=42 \mathrm{sec} . \\
& t_{H L C}=0.274 \tau_{H L L}=1.1 \mathrm{sec} . \\
& t_{L}=2.06 \times 10^{8} \mathrm{sec} .
\end{aligned}
$$

If the fracture extends as a PKN fracture, storage effects will dominate leak-off until very large times because the times $t_{S}$ and $t_{L}$ are much larger than typical pumping times for fracturing jobs. Therefore, the appropriate model to use for evaluating $t_{H L P}$ and $t_{H L C}$ is the small leak-off model. From the results computed for $t_{H L P}$ and $t_{H L C}$ from the small leak-off model we see that the aspect ratio becomes equal to unity in the neighborhood of the time range $252 \mathrm{sec}$. to $549 \mathrm{sec}$. If the transition time is smaller than the lower limit of this time range, it is possible for the pressure decline period due to CGDD-type fracture extension not to be well defined on the bottomhole pressure record in this fracturing job. However, it can be seen from Equation (4.4) that ${ }^{\mathrm{HLS}}$ increases with decreasing pumping rate, and increasing apparent fluid viscosity, and is a strong function of fracture height. Therefore, each fracture job must be separately evaluated for the existence of an initial pressure decline segment in the bottomhole pressure record. 


\section{SENSITIVITY ANALYSIS AND ERROR ESTIMATES}

\subsection{Simplifying Assumptions}

The primary motivation for undertaking this analysis is to determine the feasibility of estimating the crack-opening modulus $E_{c}$ from hydraulic fracturing bottomhole pressure records. In this section we examine under what conditions this would be possible, through analysis based on constant height models. For this purpose we use the equation:

$$
p_{w}(t)-S=p_{0} p_{D}(0) t^{p}
$$

We assume that the dimensionless pressure at the wellbore $p_{D}(0)$ and the dimensionless parameters $\alpha$ and $B$ are computed by solving the appropriate nonlinear ordinary differential equations given in this report for small leak-off and small storage limiting conditions. These quantities are in general functions of the power-law exponents $q, n^{\prime}$ and $m$ and the type of model (PKN or CGDD) being used to analyze data from a particular time regime of the fracturing process. We will pay special attention to the case of $q=0$ for a constant rate of injection of the fracturing fluid and will assume that the rheology of the fluid characterized through laboratory measured $K^{\prime}$ and $n^{\prime}$ remains valid within the fracture under reservoir conditions. Thus, we exclude the possibility of frictional, thermal and chemical degradation of the fracturing fluid. We also assume that the fluid leak-off behavior is well defined by the leak-off coefficient $\mathrm{C}$ and the exponent $\mathrm{m}$ measured in the laboratory. Finally, we assume that an accurate estimate of the minimum in-situ principal stress $S$, perhaps obtained through pump-in/flow-back tests, is available. As a result of these simplifying assumptions, only the crack-opening modulus $E_{c}$ and the fracture height $H$ remain as uncertain parameters.

In addition to the uncertainty of these parameters that are required as input to the present model there are other uncertainties that arise as a result of the modelling approximations that are embedded in the models 
themselves. These include the assumption of constant and uniform fracture height, the crack-opening stiffness assumptions, the assumption of flow between smooth parallel walls instead of flow within a rough wavy fracture, the assumption of one-dimensional pressure-independent leak-off to the formation, the neglect of the details of fluid flow and stress distribution near the crack-tip and the effects of non-uniform in-situ modulus and stress distributions. If the proppant is added to the fracturing fluid, then we must also consider the effects of proppant settling and entrapment between the fracture walls on fluid flow and fracture closure. We do not intend to either analyze or account for all of these complexities in this report, but we will consider the effect of uncertainty in fracture height on our assumptions because the assumed fracture height is one of the major uncertain parameters in constant height models.

\subsection{Bottomhole Pressure Time Response of PKN and CGOD Fractures}

Let us first examine the effect of the PKN and CGDO model assumptions on the time history of the bottomhole pressure. To present the results for both types of fractures on the same plot Equation (5.1) is recast in the form:

$$
\frac{p_{W}(t)-S}{p_{0} p_{D}(0) \tau^{p}}=t_{D}^{p} ; \quad t_{D}=t / \tau \text {, }
$$

where $\tau=\tau_{P}$ and $\tau_{C}$ for PKN and CGOD fractures, respectively. This relationship is plotted in Figure 3 for PKN and CGDD fractures for the small leak-off and small storage limiting conditions and for parameter values of $q=0, m=1 / 2$ and $n^{\prime}=1,1 / 2$. As expected, the bottomhole pressure grows with time for PKN fractures while it decays with time for CGDD fractures. Both models are sensitive to the fluid power-law index $n$ ' in the small leak-off and the small storage limits. The trend for PKN fractures is towards higher pressures with increasing non-Newtonian behavior (i.e. decreasing $n^{\prime}$ ) of the fracturing fluid. An opposite trend with lower bottomhole pressures is exhibited by CGDD fractures for increasingly non-Newtonian fracture fluids. Also, the effect of 


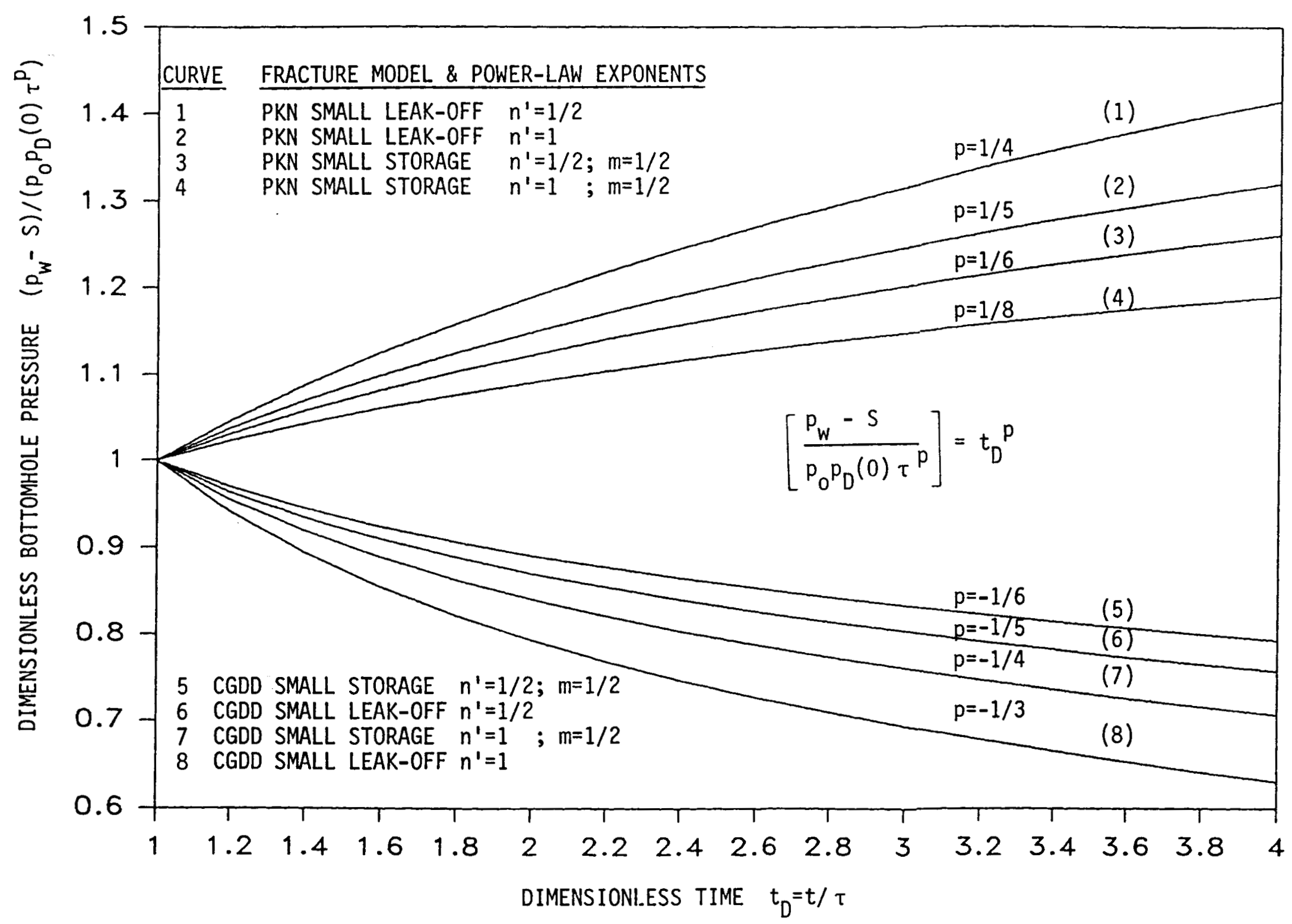

FIGURE 3: BOTTOMHOLE PRESSURE HISTORY FOR PKN AND CGDD CONSTANT HEIGHT FRACTURES. 
neglecting fluid leak-off while retaining the fluid storage term on PKN fractures is opposite to that for CGOD fractures. In PKN fractures, neglect of fluid leak-off for constant flow rate injection increases the bottomhole pressure. For CGDD fractures, neglect of fluid leak-off results in lower bottomhole pressures. These differences result from the fact that fracture length is directly coupled through the crack-opening relation to the pressure distribution in CGDD fractures whereas this coupling is only through the fluid mass conservation equation in the case of PKN fractures.

As previously stated, a time period during which the bottomhole pressure increases at a rate consistent with stable fracture extension at constant height predicted by PKN models is often observed in pressure records. If the in-situ minimum principal stress $S$ is known, then $\left(p_{w}(t)-s\right)$ can be computed from the bottomhole pressure data and plotted against time on a $\log -\log$ plot. From Equation (5.1) we see that:

$$
\ln \left\{p_{w}(t)-S\right\}=\ln \left\{p_{0} p_{D}(0)\right\}+p \ln \{t\}
$$

so that the pressure power-law exponent $p$ and the product $\left\{p_{0} p_{D}(0)\right\}$ can be obtained from the slope and intercept, respectively, of the log-log plot. A procedure for extracting the value of the crack-opening modulus $E_{c}$ can, therefore, be based on first using the value of $p$ thus determined to select the appropriate power-law model for analysis and then using the value of the product $\left\{p_{0} p_{D}(0)\right\}$ to determine the value of the crack-opening modulus $E_{c}$ from the results for the power-law coefficient for that model. Note that, as previously stated, this assumes that the necessary calculations have been performed to determine $\alpha, B$ and $p_{D}(0)$ for each model as a functions of $q, n^{\prime}$ and $m$.

\subsection{Sensitivity of Bottomhole Pressure Coefficient To Crack-Opening Modulus And Fracture Height}

For any two sets of the parameters governing the hydraulic fracturing process the uncertainty of the power-law pressure coefficients $\rho_{0}$ can be calculated from Equations (3.52), (3.56), (3.62) and (3.66). If it is assumed, for convenience, that there is no uncertainty in any parameters 
other than $E_{c}$ and $H$, the following results are obtained from the different models for the sensitivity of the pressure coefficients $p_{0}$ to these parameters:

\subsubsection{Case of Small Fluid Leak-off}

\section{PKN FRACTURES}

$\left(p_{02} / p_{01}\right)=\left(E_{c 2} / E_{c 1}\right)^{\left(2 n^{\prime}+2\right) /\left(2 n^{\prime}+3\right)}\left(H_{2} / H_{1}\right)^{-\left(3 n^{\prime}+3\right) /\left(2 n^{\prime}+3\right)}$,

$P_{0} \sim E_{c}{ }^{e} ; \quad 2 / 3 \leq e \leq 4 / 5 ; \quad 0 \leq n^{\prime} \leq 1 \quad$ for fixed $H$,

$p_{0} \sim H^{h} ; \quad-1 \leq h \leq-6 / 5 ; \quad 0 \leq n^{\prime} \leq 1$ for fixed $E_{c} \cdot$

\section{CGDD FRACTURES}

$\left(p_{02} / p_{01}\right)=\left(E_{c 2} / E_{c 1}\right)^{\left\{\left(n^{\prime}+1\right) /\left(n^{\prime}+2\right)\right\}}$,

$P_{0} \sim E_{c}^{e} ; \quad 1 / 2 \leq e \leq 2 / 3 ; \quad 0 \leq n^{\prime} \leq 1$ for fixed $H$,

\subsubsection{Case of Small Fluid Storage}

\section{PKN FRACTURES}

$\left(p_{02} / p_{o 1}\right)=\left(E_{c 2} / E_{c 1}\right)^{\left\{\left(2 n^{\prime}+1\right) /\left(2 n^{\prime}+2\right)\right\}}\left(H_{2} / H_{1}\right)^{-\left(3 n^{\prime}+2\right) /\left(2 n^{\prime}+2\right)}$,

$P_{0} \sim E_{c}{ }^{e} ; \quad 1 / 2 \leq e \leq 3 / 4 ; \quad 0 \leq n^{\prime} \leq 1$ for fixed $H$,

$\rho_{0} \sim H^{h} ; \quad-1 \leq h \leq-5 / 4 ; \quad 0 \leq n^{\prime} \leq 1$ for fixed $E_{c}$. 


\section{CGDD FRACTURES}

$$
\begin{aligned}
& \left(p_{02} / p_{01}\right)=\left(E_{c 2} / E_{c 1}\right)^{\left(2 n^{\prime}+1\right) /\left(2 n^{\prime}+2\right)}\left(H_{2} / H_{1}\right)^{n^{\prime} /\left(2 n^{\prime}+2\right)}, \\
& p_{0} \sim E_{c}^{e} ; \quad 1 / 2 \leq e \leq 3 / 4 ; \quad 0 \leq n^{\prime} \leq 1 \text { for fixed } H \text {, } \\
& P_{0} \sim H^{h} ; \quad-1 \leq h \leq-1 / 4 ; \quad 0 \leq n^{\prime} \leq 1 \text { for fixed } E_{c} \text {. }
\end{aligned}
$$

The sensitivity of the pressure coefficient $P_{0}$ to variation of the crack-opening modulus $E_{c}$ and the assumed fracture height $H$ can be assessed from Equations (5.4) to (5.7). The variation of $p_{02} / p_{01}$ as either the crack-opening modulus $E_{c}$ or the fracture height $H$ vary over a factor of four are graphed in Figures 4 and 5 for the values of $n^{\prime}=1$ and $n^{\prime}=1 / 2$. A factor of two uncertainty in the crack-opening modulus alone results in approximately a factor of 1.5 uncertainty in the pressure coefficient ratio for all models. At greater values of modulus uncertainty, the differences in the models leads to larger or smaller uncertainties with model type and the power-law index $n$ '. For PKN and CGOD models, the value of $n^{\prime}$ (chosen to be $1 / 2$ or 1 ) has a greater impact on the sensitivity of the pressure coefficient ratio than the choice of the small leak-off or small storage approximation. These results are useful for judging how sensitive the pressure record would be to the in-situ crack-opening modulus and how much of a difference in the measurements we can expect for a given uncertainty in this parameter.

\subsection{Uncertainty in the Calculated Crack-Opening Modulus Due to Uncertainty in Fracture Height}

While the above results indicate how sensitive the pressure response would be to the crack-opening modulus and fracture height, the sensitivity of the crack-opening modulus, computed from a given pressure record, to the choice of the model used to interpret the pressure data and to the assumed fracture height is of greater interest. To evaluate this, we set $p_{02}=p_{01}$ in Equations $(5.4 a),(5.5 a),(5.6 a)$ and (5.7a) to obtain: 


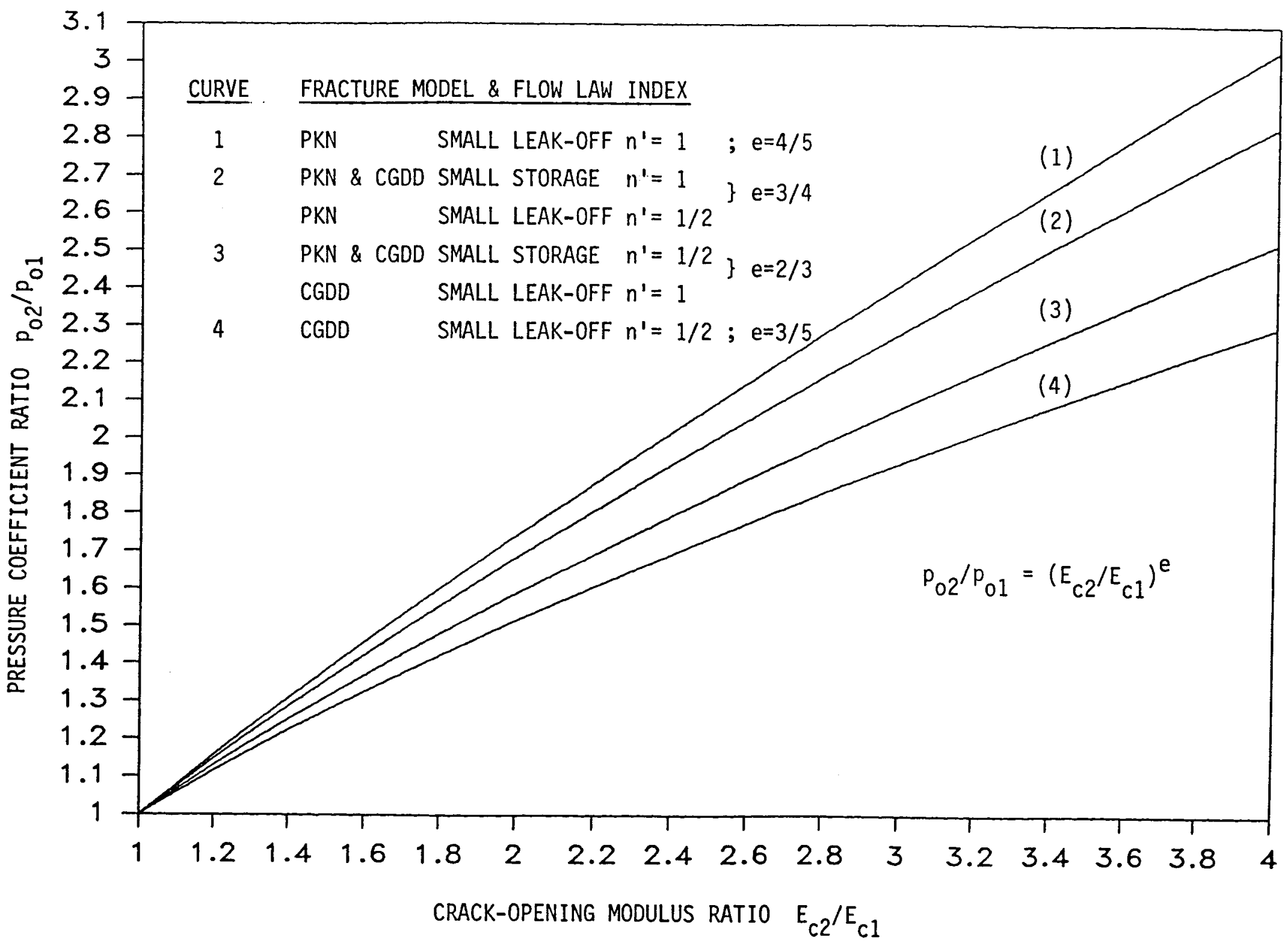

FIGURE 4: SENSITIVITY OF BOTTOMHOLE PRESSURE COEFFICIENT $p_{0}$ TO CRACK-OPENING MODULUS $E_{c}$. 


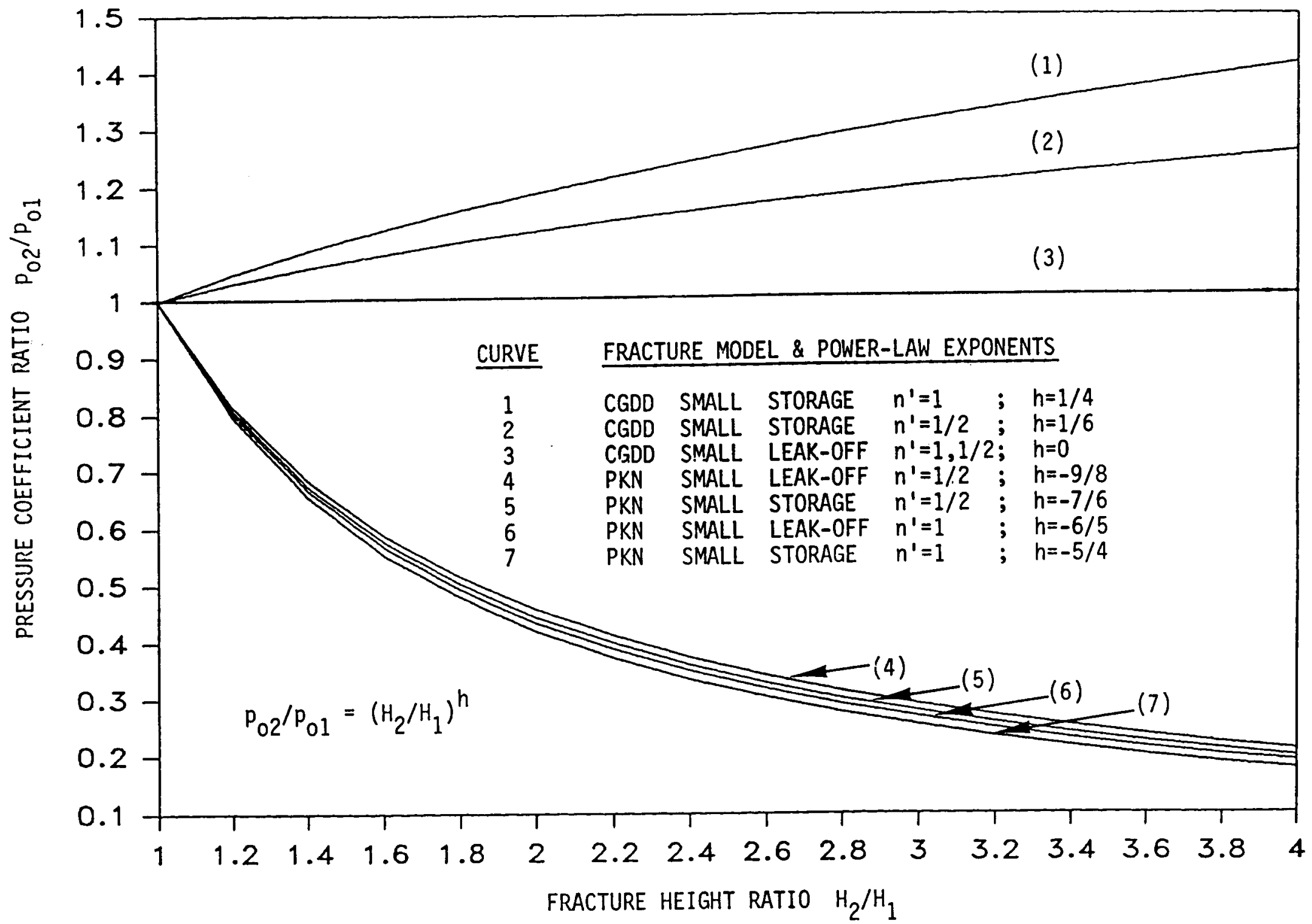

FIGURE 5: SENSITIVITY OF BOTTOMHOLE PRESSURE COEFFICIENT $p_{0}$ TO FRACTURE HEIGHT H. 


\subsubsection{Case of Small Fluid Leak-Off}

PKN FRACTURES

$$
\begin{gathered}
\left(E_{c 2} / E_{c 1}\right)=\left(H_{2} / H_{1}\right)^{3 / 2}, \\
\text { CGDD FRACTURES } \\
\left(E_{c 2} / E_{c 1}\right)=1
\end{gathered}
$$

\subsubsection{Case of Small Fluid Storage}

$$
\begin{aligned}
& \text { PKN FRACTURES } \\
& \left(E_{c 2} / E_{c 1}\right)=\left(H_{2} / H_{1}\right)^{\left(3 n^{\prime}+2\right) /\left(2 n^{\prime}+1\right),} \\
& E_{c} \sim H^{h} ; \quad 2 \geq h \geq 5 / 4 ; \quad 0 \leq n^{\prime} 1 . \\
& \text { CGDD FRACTURES } \\
& \left(E_{c 2} / E_{c 1}\right)=\left(H_{2} / H_{1}\right)^{-n^{\prime} /\left(2 n^{\prime}+1\right)}, \\
& E_{c} \sim H^{h} ; \quad 1 / 2 \leq h \leq 3 / 4 ; \quad 0 \leq n^{\prime} \leq 1,
\end{aligned}
$$

The sensitivity of the crack-opening modulus $E_{c}$ to variation of the assumed fracture height $H$ can be assessed from Equations (5.8) through (5.11) and is plotted in Figure 6 for $n^{\prime}=1$ and $1 / 2$. It is seen that the dominant difference is in the model used to interpret the data. For CGDO models, the computed crack-opening modulus is relatively insensitive to fracture height, a feature that can be exploited to our advantage if the fracturing job is designed to yield a well defined period of CGDD-type fracture extension at early times. The crack-opening modulus computed 
from the PKN model, on other hand, is very sensitive to fracture height and yields a three-fold increase in modulus for a two-fold increase in assumed fracture height. The PKN model for both small leak-off and small storage conditions is equally sensitive to fracture height at uncertainty ratios less than 2, but this difference increases with increasing fracture height uncertainty ratio. The difference between PKN models for small leak-off and for small storage is generally greater than the differences due to the non-Newtonian power-law exponent $n^{\prime}$ over the range $1 / 2$ to 1 . The effect of non-Newtonian fracturing fluid behavior is to increase the uncertainty in modulus when the PKN small storage modulus is used. The effect on the uncertainty in the modulus with change in $n^{\prime}$ is not significant for CGOD fractures.

Because many bottomhole pressure records exhibit a time period during which the bottomhole pressure response is consistent with stable PKN-type fracture extension at constant height, the high degree of sensitivity of the crack-opening modulus to the assumed fracture height is unfortunate. As can be seen from figure 6 even a $50 \%$ uncertainty in fracture height during this time period will lead to an error of $100 \%$ in the crack-opening modulus with the error doubling to $200 \%$ if the fracture height is in error by $100 \%$. The uncertainty in fracture height can arise from many causes. For example, the fracture height can be uncertain due to vertical growth of the fracture during its extension because in-situ stress and modulus barriers that favor containment of the fracture do not exist. On the other hand, a fracture may be well contained by such barriers but the height at which it is contained could be uncertain because the locations of the barriers themselves relative to the perforations are uncertain. Another possibility is that the perforated height at the wellbore is much smaller than the barrier height so that substantial equidimensional fracture growth will occur before the barriers are reached.

A variety of different analyses similar to the present sensitivity study can be performed using the basic relationships derived in Section 3 , by making different choices of the uncertain variables. These choices are best guided by the specific fracturing conditions prevailing in the field. 


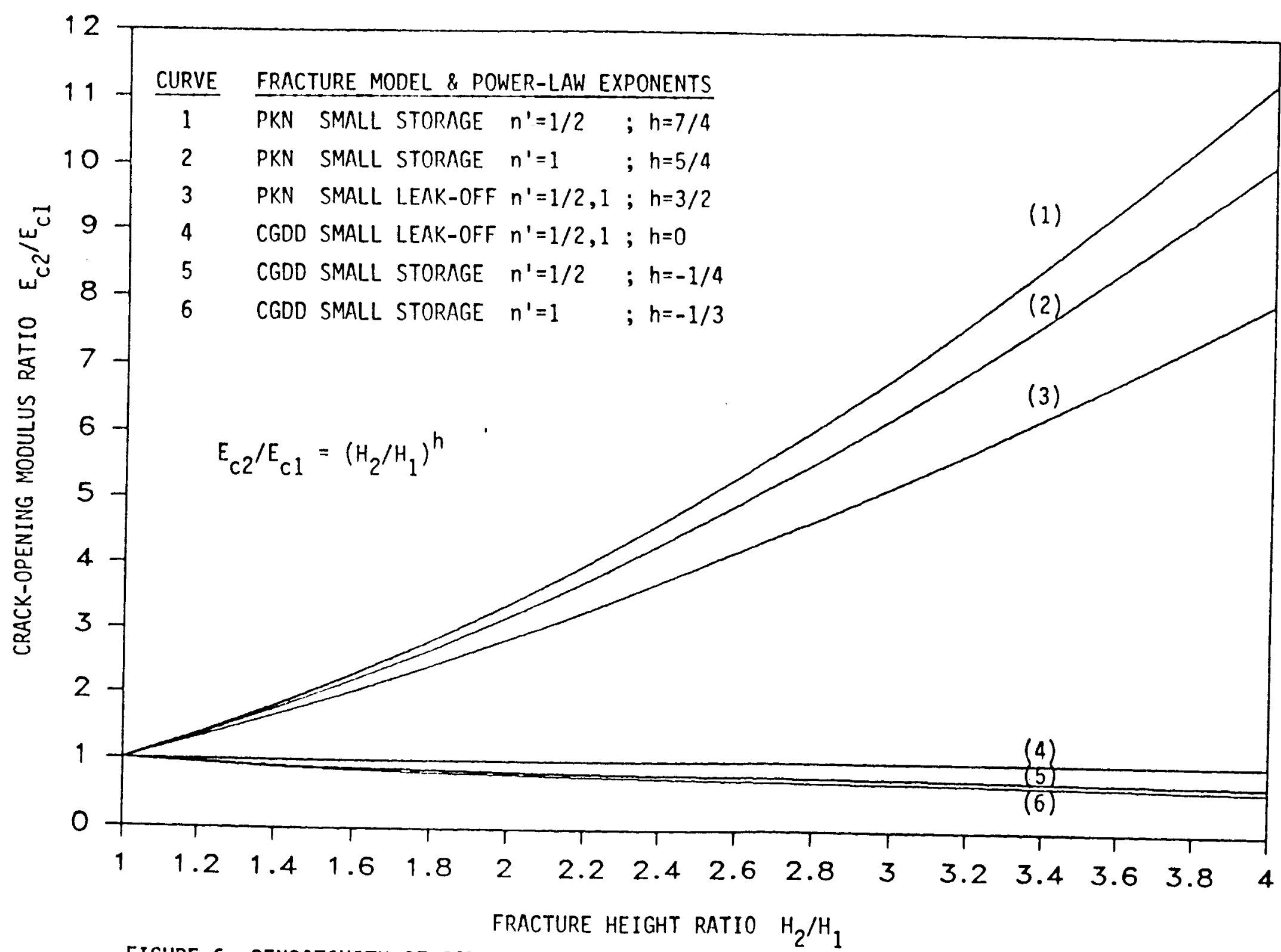

FIGURE 6: SENSITIVITY OF CALCULATED CRACK-OPENING MODULUS $E_{c}$ TO ASSUMED FRACTURE HEIGHT $H$. 
We have focused on the fracture height problem because, in most situations, the conditions are not favorable to containment of the fracture at a uniform and constant height. However, up to a certain point that depends on the size of the fracture to be created, it is possible to choose the fracturing fluid rheology, the pumping rate and the location of the perforations relative to barriers to prolong the containment of the fracture by any existing barriers. Ultimately, if the pumping is continued long enough at a sufficiently high rate, the pressure at the wellbore will become large enough to overcome any existing favorable stress contrasts and modulus barriers and height growth will occur. Whenever significant height growth occurs, analyses based on constant height fracture models must be abandoned in favor of models that allow simultaneous growth of the fracture in the longitudinal and lateral directions. 


\section{SUMMARY AND CONCLUSIONS}

In this report the analysis of constant height PKN and CGDD types of models for hydraulic fracturing of underground rock formations was brought within a unified theoretical framework. A relatively transparent, but rigorous, similarity analysis of the governing equations was used to extract the time dependence of the solutions for the fluid pressure, fracture width, fluid flow rate and fracture length of hydraulic fractures created by fluid injection at the wellbore at a rate that varies as a power of time. To obtain the distribution of each of these quantities over the fracture it is necessary to solve the nonlinear ordinary differential equation that governs the similarity solution. This task is beyond the scope of the present effort but must be undertaken to complete these solutions. The growth laws that result from this analysis provide a systematic means for assessing the differences, limitations and domains of applicability of constant height fracture models (PKN and CGDD), and for evaluating their sensitivity to the physical parameters that govern hydraulic fracturing. Furthermore, as a natural outcome of this unified approach, it has been possible to develop an extended view of constant height fractures as a hybrid of CGDD fractures at small aspect ratios that evolve into PKN fractures at large aspect ratios. The power-law similarity solutions were used to devise a procedure to determine the crack-opening modulus from bottomhole pressure records. Results of the model development results and of the sensitivity study on the estimation of the crack-opening modulus from bottomhole pressure data are summarized below.

\subsection{Modelling Results}

The special features and results given in this report for the PKN, CGDO, and hybrid constant height fracture models are:

o Cross-sections of arbitrary shape which are allowed through the shape factors $Y_{W}$ and $Y_{Q}$. 
- Non-Newtonian power-law rheology of the fracturing fluid that is characterized by $K^{\prime}$ and $n^{\prime}$.

- Fluid leak-off that varies as an arbitrary power $m$ of the elapsed time.

o Fluid injection rate that varies as an arbitrary power q of time.

- Power-law coefficients and power-law exponents for fracture fluid pressure, fracture width, fracture fluid flow and fracture length.

- Estimates provided for the times $t_{S}$ and $t_{L}$ that define the $t i m e$ regimes when the small leak-off and small storage assumptions can be invoked.

o Presentation of an extended view of constant height models as a hybrid of CGOD and PKN models.

- Estimates provided for the times $t_{H L P}$ and $t_{H L C}$ that define the transition between CGDD-like and PKN-like fracture behavior.

o Sensitivity analysis of the effect of fracture height on crack-opening modulus computed from bottomhole pressure data.

Because constant height fracture models have been the subject of considerable previous work it is appropriate to briefly identify here where the present work extends previously reported results. The complete solution to the similarity equations for PKN fractures was given by Nordgren [28] for the special case of a Newtonian fluid, the half-power fluid leak-off law and constant fluid injection rate. Cleary [6] has presented the most general similarity solutions for PKN fractures with non-Newtonian power-law fluids, generalized power-law fluid leak-off and power-law fluid injection rates. However, as in the present work, no rigorous computational results comparable to Nordgren's work were given by him. Geertsma and De Klerk [9] provided an approximate solution for CGDD fractures that neglects the fluid storage term and approximates the 
crack-opening relationship. Their work is limited to Newtonian fluid flow in the fracture, half-power fluid leak-off to the rock formation and a constant rate of fluid injection. Daneshy [7] has reported an approximate extension of this model to power-law fracturing fluids but information is not available in sufficient detail regarding this improvement.

In summary, a general theory has been presented by Cleary for PKN fractures but no rigorous computations of the similarity equations are available. For CGDD fractures, no similarity analysis that accounts for all of the features included in the model presented in this document has been previously reported in the literature. With the exception of the results reported by Nordgren for a special case of PKN fractures, the extended treatment of constant height fractures as a hybrid of CGDD fractures at small aspect ratios that evolve into PKN fractures at large aspect ratios has not been reported previously. In particular, with the exception of $\tau_{p}$ that is embedded in Nordgren's work, estimates of the characteristic times ' ${ }_{P}$ ' ' ${ }$ ' ${ }^{\prime}{ }_{H L S}$ and ' ${ }_{H L P}$ and the transition times $t_{S}, t_{L}, t_{H L P}$ and $t_{H L C}$ have not been previously identified for PKN and CGDD fractures or for the newly proposed hybrid extended CGOD-PKN model for constant height fractures.

\subsection{Estimation of Crack-Opening Modulus}

If the hydraulic fracture is well contained and extends stably at constant height, then the similarity power-law growth expressions derived here for the bottomhole pressure provide a simple means of computing the crack-opening modulus from the bottomhole pressure history. Under these conditions:

- The fracture will extend initially as a CGDD-type fracture at small aspect ratios $(L(t)<<1)$ and evolve into a PKN-type fracture at large aspect ratios $(L(t) / H>>1)$. Depending on the fracturing conditions both of these regimes may not be observed. The time estimates that are given in this report provide a means of designing 
for a period of stable fracture extension that will exhibit at least one well defined segment of the bottomhole pressure record that can be analyzed using the expressions given here for the power-law coefficient and power-law exponent of the bottomhole pressure for PKN and CGDD fractures.

- The calculated crack-opening modulus values are sensitive to the choice of model used to analyze the data. If the bottomhole pressure decays with time, a CGOD-type model should be used to analyze the data provided the time estimates indicate that the use of the CGDD model is valid. Similarly, if the bottomhole pressure increases with time the PKN model should be used provided the time estimates indicate that this is appropriate. In both cases, comparison of the measured and computed bottomhole pressure power-law exponent will serve as an independent check on the validity of the analysis.

o Subject to the modelling approximations and the assumption that there is no uncertainty in the fluid properties, the uncertainty in the crack-opening modulus will be mostly due to the uncertainty in the assumed fracture height. The crack-opening modulus computed from the CGOD fracture model is insensitive to the choice of fracture height, while the opposite is true for PKN-type fracture extension. Therefore, CGDD-type extension is preferable to PKN-type fracture extension for determining the crack-opening modulus, and should be considered to be a design goal for the purpose of determining the crack-opening modulus. If the PKN-type model is the model that must be used to compute the crack-opening modulus, then it should be recognized that fracture height must be known with greater accuracy than the accuracy with which we desire to measure the crack-opening modulus.

- If fracture height is significantly nonuniform or growing in time, then the results given here do not hold and variable height models must be used for analyzing the bottomhole pressure data. Height growth is pervasive; it cannot be completely suppressed and is significant at 
even moderately favorable stress contrasts at the stress barriers. Therefore, to conduct a systematic study of the impact of height growth on the bottomhole pressure and of the sensitivity of the model assumptions on the computed crack-opening modulus a reliable variable height simulator, preferably belonging to the third generation of models, would be required. 


\section{NOMENCLATURE}

\section{English Symbols}

\begin{tabular}{|c|c|}
\hline & - Fluid leak-off coefficient (Equation (2.24a)) \\
\hline & - Fluid density (Equation (2.3)) \\
\hline & - Crack-opening modulus exponent (Section 5.2) \\
\hline & - Crack-opening modulus $\mathrm{G} /(1-v)$ \\
\hline & - Cross-sectional shape function (Equation (2.6)) \\
\hline & - Fracture height exponent (Section 5.2) \\
\hline & - Height of the fracture \\
\hline & - Leak-off height of the racture \\
\hline & - Fracture toughness (Equation (2.22b)) \\
\hline & - Power-law fluid consistency (Equation (2.12)) \\
\hline & $\begin{array}{l}\text { - Apparent fluid viscosity of power-law fluid (Equation (2.17)) } \\
\text { - Fracture length power-law growth exponent (Equation (3.2)) }\end{array}$ \\
\hline & $\begin{array}{l}\text { - Fracture length power-law growth exponent } \\
\text { for CGDD fractures (Equation ( } 4.5 \mathrm{~b}) \text { ) }\end{array}$ \\
\hline & $\begin{array}{l}\text { - Fracture length power-law growth exponent } \\
\text { for PKN fractures (Equation ( } 4.4 \mathrm{~b}) \text { ) }\end{array}$ \\
\hline & - Length of the fracture \\
\hline & - Dimensionless fracture length $L(t) / H$ \\
\hline & $\begin{array}{l}\text { - Leak-off power-law exponent (Equation (2.24a)) } \\
\text { - Power-law fluid rheology index (Equation (2.12)) }\end{array}$ \\
\hline t) & - Fluid pressure in the fracture \\
\hline & - Average fluid pressure in the fracture \\
\hline & - Dimensionless fluid pressure in the fracture (Equation (3.3)) \\
\hline & - Pore fluid pressure in the far-field \\
\hline & - Fluid pressure power-law coefficient (Equation (3.3)) \\
\hline & - Flowing bottomhole fluid pressure (Equation (3.30)) \\
\hline & - Flowrate power-law growth exponent (Equation (3.5)) \\
\hline$x_{0}$ & $\begin{array}{l}\text { - Fluid leak-off rate (at fracture cross-section) } \\
\text { (Equation (2.5)) }\end{array}$ \\
\hline
\end{tabular}


${ }^{\mathrm{L}} \mathrm{LD}$

$q_{\text {Lo }}$

$q_{x}(x, z, t)$

$Q(x, t)$

$Q_{D}$

$Q_{0}$

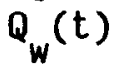

$\mathbf{r}$

$\mathrm{S}$

$\mathrm{t}$

$t_{D}$

$t_{D L}$

tDS

$t_{H L C}$

$t_{\text {HLP }}$

$t_{L}$

$\mathrm{t}_{\mathrm{S}}$

$v_{x}, v_{y}, v_{z}$

$w(x, z, t)$

$w(x, t)$

$w_{0}$

$x, y, z$
- Dimensionless fluid leak-off (Equation (3.6))

- Fluid leak-off rate power-law coefficient (Equation (3.6))

- Flow rate in $x$-direction across fracture width (Equation (2.15))

- Flow rate in $x$-direction across fracture cross-section (Equation (2.4))

- Dimensionless fluid flow rate (Equation (3.5))

- Flow rate power-law coefficient (Equation (3.5))

- Flow rate into the fracture at the wellbore (Equation (3.7))

- Fluid leak-off power-law growth exponent (Equation (3.6))

- Minimum in-situ principal stress assumed to act normal to the fracture plane

- time

- Dimensionless time (Equation (5.2))

- Dimensionless time $t / \tau_{\text {HLS }}$ for small storage large leak-off condition (Section 4.1.1)

- Dimensionless time $t / \tau_{H L L}$ for small leak-off condition (Section 4.1.1)

- Aspect ratio limiting time for CGDD fractures (Equations (4.10), (4.12))

- Aspect ratio limiting time for PKN fractures (Equations (4.9), (4.11))

- Limiting time for small fluid storage assumption (Equation (3.70a))

- Limiting time for small fluid leak-off assumption (Equation (3.71a))

- Fracture fluid velocity components in the $x, y$ and $z$ coordinate directions

- Fracture width profile at a cross-section (Equation 2.6))

- Fracture width at the center of each cross-section (Equation (2.6))

- Dimensionless fracture width (Equation (3.4))

- Fracture width power-law coefficient (Equation (3.4))

- Spatial coordinates in the longitudinal (horizontal), normal and lateral (vertical) directions with respect to the fracture 


\section{Greek Symbols}

$\alpha$

B

$r$
$r$
$r$
$r$

$v$

$\boldsymbol{\xi}$

$\rho$

$\sigma$

$T(x)$

$\tau_{C}$

$\tau_{p}$

$\tau_{\text {HLS }}$

$T_{\text {HLL }}$

- Parameter that measures the relative importance of fluid storage and fluid transport effects (Equation 3.21a))

- Parameter that measures the relative importance of fluid leak-off and fluid transport effects (Equation (3.21b)

- Ratio of the fluid leak-off height to fracture height

- Flow rate shape parameter (Equation (2.18))

- Fracture width shape parameter (Equation (2.7))

- Dummy integration variable

- Dimensionless similarity variable (Equation (3.1))

- Poisson's Ratio

- Dummy integration variable

- Average fluid density at a cross-section Equation (2.3))

- Specific fracture surface energy (Section 2.3)

- Time of arrival of fracture front at location $x$ (Equation (2.24a))

- Characteristic time for extension of CGDD fractures (Equation 3.55b))

- Characteristic time for extension of PKN fractures (Equation (3.59b))

- Characteristic time for extension of PKN and CGDD fractures under small leak-off large storage conditions (Equation (4.3))

- Characteristic time for extension of PKN and CGOD fractures under small storage large leak-off conditions (Equation (4.6))

- Fluid shear stress on the fracture surface (Equation (2.12))

- Fracture width power-law growth exponent (Equation (3.4)) 


\section{REFERENCES}

1. Abou-Sayed, A.S., Breckett, C.E. and Clifton, R.J.:"In-Situ Stress Determination by Hydrofracturing-A Fracture Mechanics Approach, "]. Geophys. Res., V.83,B6,pp. 2851-2862, 1978.

2. Abou Sayed, A.S., Sinha, K.P. and Clifton, R.J.:"Evaluation of the Influence of In-Situ Reservoir Conditions on the Geometry of Hydraulic Fractures Using a 3-D Simulator: Part 2 - Case Studies," Paper SPE/DOE/GRI 12878 presented at the SPE/DOE/GRI Unconventional Gas Recovery Symp., Pittsburgh, PA., May 1984.

3. Advani, S.H., Torok, J.S. and Lee, J.K.:"Explicit Approximate Solutions Associated With the Mechanics of Hydraulic Fracturing, "J. App. Mech., Trans. ASME., (In Press).

4. Barenblatt, G.I.:"The Mathematical Theory of Equilibrium Cracks in Brittle Fracture," Advances in Applied Mechanics, Ed. Dryden H.L and Von Karman, Th., V. 7,p. 56, Academic Press, NY., 1962.

5. Booker, J.R.:"Time Dependent Strains Following Faulting of a Porous Medium," J. Geophys. Res.,V 79, pp. 2037-2044, 1974.

6. Cleary, M.P.:"Comprehensive Design Formulae for Hydraulic Fracturing," Paper SPE 9259 presented at the 55th Annual Fall Technical Conference of the Soc. Pet. Engrs., Dallas, TX, September 21-24, 1980.

7. Daneshy, A.A.:"On the Design of Vertical Hydraulic Fractures," Tech., pp. 83-97, January 1973.

8. England, A.H. and Green, A.E.: "Some Two-Dimensional Punch and Crack Problems in Classical Elasticity," Proc. Cambridge Phil. Soc., V.59, pp. $489-500,1963$.

9. Geertsma, J. and De Klerk, F.:"A Rapid Method of Predicting Width and Extent of Hydraulic Induced Fractures," J. Pet. Tech.,pp. 1571-1581, December 1969.

10. Geertsma, J. and Haafkens, R.: "A Comparision of the Theories for Predicting Width and Extent of Vertical Hydraulically Induced Fractures," J. Energy Resources Tech., Trans. ASME., pp. 8-19, V.101, March 1979.

11. Haimson, B. and Fairhurst C.: "In-Situ Stress Determination At Great Depth by Means of Hydraulic Fracturing," in Rock Mechanics-Theory and Practice, Ed. W.H. Somerton, pp. 559-584, Soc. of Mining Engineers, American Inst. of Mining, Met. and Pet. Engrs, Inc., Salt Lake City, 1970.

12. Heuze, F.E.: "Scale Effects in the Determination of Rock Mass Strength and Deformability," Rock Mechanics, V.12,pp. 167-192, 1980.

13. Howard, G.C. and Fast, C.R.: Hydraulic Fracturing, Monograph Vol. 2, Soc. Pet. Engrs., 1970. 
14. Hubbert, M.K. and Willis, D.G.: "Mechanics of Hydraulic Fracturing," Irans. AIME, V.210,pp. 153-166, 1957.

15. Keller, D.A., Davis, A.H. and Stewart, J.N.:"The Calculation of Steam Flow and Hydraulic Fracturing in a Porous Medium with the KRAK Code," Los Alamos Scientific Laboratories, LA-5602-MS, 1974.

16. Khristianovitch, S.A. and Zheltov, Y.P.:"Formation of Vertical Fractures by Means of Highly Viscous Fluid," Proc. Fourth World Pet. Congr., pp. 579-586, Section II, Carlo Colombo, Rome 1955.

17. Lama, R.D. and Vutukuri, V.S.: Handbook on Mechanical Properties of Rocks, Volume IV-Testing Techniques and Results, Trans Tech Publications, Clausthal, Germany, 1978.

18. Lin, W.:" Strength and Static Elastic Moduli of Mesaverde Rocks," Lawrence Livermore National Laboratory, UCID-20370, March 1985.

19. Lin, W.:"Ultrasonic Velocities and Dynamic Elastic Moduli of Mesaverde Rocks," UCID-20273 Rev. 1, March, 1985.

20. Lin, W. and Heuze, F.E.:"In-Situ Dynamic Elastic Moduli of Mesaverde Rocks, and a Comparision with Static and Dynamic Laboratory Moduli," Lawrence Livermore National Laboratory, UCID-20611, January 1986.

21. Loitsyanskii, L.G.: Mechanics of Liquids And Gases, Pergamon Press, New York, NY., 1966.

22. Mendelsohn, D.A.: "A Review of Hydraulic Fracture Modeling-Part I: General Concepts, 2D Models, Motivation for 3D Modeling," J. Energy Resources Tech., Trans. ASME., V. 106, pp. 369-376, September, 1984.

23. Mendelsohn, D.A.:"A Review of Hydraulic Fracture Modeling-II: 3D Modeling and Vertical Growth in Layered Rock," ]. Energy Resources Tech., Trans. ASME.,V. 106,pp. 543-553, December, 1984 .

24. Morita, N., Whitfill, D.L. and Wahl, H.A.:"Stress-Intensity Factor and Fracture Cross-Sectional Shape Predictions From a 3-D Model for Hydraulically Induced Fractures," Paper SPE 14262 presented at the 60th Ann. Mtg. of the Soc. Pet. Engrs., Las Vegas, NV., September 22-25, 1985.

25. Nemat-Nassèr, S., Abe, H. and Hirakawa, S. Eds. Hydraulic Fracturing and Geothermal Energy, Martinus Nijhoff, 1983.

26. Nolte, K.G. and Smith, M.B.:" Interpretation of Fracturing Pressures," J. Pet. Tech., pp.1967-1775, September 1981.

27. Nolte, K.G.:" Fracture Design Considerations Based on Pressure Analysis," Paper SPE 10911 presented at the 1982 Soc. Pet. Engrs. Cotton Valley Symposium, Tyler, TX, May 20, 1982. 
28. Nordgren, R.P.:"Propagation of a Vertical Hydraulic Fracture," Soc. Pet. Engrs. J., pp. 306-314, August 1972.

29. Nur, A. and Booker, J.R.:"Aftershocks Caused by Fluid Flow," Science, V.175, p.885, 1972.

30. Perkins, T.K. and Krech, W.W,:" The Energy Balance Concept of Hydraulic Fracturing," Soc. Pet. Engrs. J.,pp. 1-12, March 1968.

31. Perkins, T.K. and Kern, L.R.: "Widths of Hydraulic Fractures," J. Pet. Tech.,pp. 937-949, September 1961.

32. Pitts, J.H. and Brandt,H: "Gas Flow in a Permeable Earth Formation Containing a Crack," J. Appl. Mech., V. 44,pp.553-558, 1977.

33. Stewart, G.E.: "Hydraulic Fracturing of Crystalline Rocks Stimulates Two Test Wells Drilled in New Hampshire," Ground Water, V.12, 1974.

34. Stout, R.B.:" Three-Dimensional Model of the Hydrofracture Process With a Rate-Dependent Variational Principle," Report No. UCID-20146, Lawrence Livermore National Laboratory, Livermore, CA., August 1984.

35. Veatch, R.W. and Crowell, R.F.:"Joint Research/Operations Programs Accelerate Massive Hydraulic Fracturing Technology," J. Pet. Tech. ,V.34,pp. 2763-2775, 1982.

36. Wolff, R. et. al.: "Stress Determination by Hydraulic Fracturing in Subsurface Waste Injection," J. Am. Water Works Assoc., V.67, 1975.

37. Zheltov, Yu. P. and Khristianovitch, S.A.:"The Hydraulic Fracturing of an Oil-Producing Formation," Izvest. Akad. Nauk SSSR, Otdel Tekh Nauk, p. 41, No. 3, 1955. 


\section{ACKNOWLEDGMENTS}

This work was performed as a part of the LLNL Unconventional Gas . Program for the Morgantown Energy Technology Center, Morgantown, WV., under Contract $W-7405-E N G-48$ with the U.S. Department of Energy.

I thank F. E. Heuze, R. B. Stout and J. L. Yow, Jr. for their helpful reviews of this document. Also, I would like to thank L. Grabowski for typing this report with her characteristic excellence. 
$-70-$

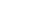

